\title{
Enhancing oscillations in intracranial electrophysiological recordings with data-driven spatial filters
}

\author{
Natalie Schaworonkow ${ }^{\mathrm{a}, *}$, Bradley Voytek ${ }^{\mathrm{a}, \mathrm{b}, \mathrm{c}, \mathrm{d}}$ \\ ${ }^{a}$ Department of Cognitive Science \\ ${ }^{b}$ Halıcioğlu Data Science Institute \\ ${ }^{c}$ Neurosciences Graduate Program \\ ${ }^{d}$ Kavli Institute for Brain and Mind, University of California, San Diego, La Jolla, CA, USA
}

\begin{abstract}
In invasive electrophysiological recordings, a variety of neuronal oscillations can be detected across the cortex, with overlap in space and time. This overlap complicates measurement of neuronal oscillations using standard referencing schemes, like common average or bipolar referencing. Here, we illustrate the effects of spatial mixing on measuring neuronal oscillations in invasive electrophysiological recordings and demonstrate the benefits of using data-driven referencing schemes in order to improve measurement of neuronal oscillations. We discuss referencing as the application of a spatial filter. Spatio-spectral decomposition is used to estimate data-driven spatial filters, a computationally fast method which specifically enhances signal-to-noise ratio for oscillations in a frequency band of interest. We show that application of these data-driven spatial filters has benefits for data exploration, investigation of temporal dynamics and assessment of peak frequencies of neuronal oscillations. We demonstrate multiple use cases, exploring between-participant variability in presence of oscillations, spatial spread and waveform shape of different rhythms as well as narrowband noise removal with the aid of spatial filters. We find high between-participant variability in the presence of neural oscillations, a large variation in spatial spread of individual rhythms and many non-sinusoidal rhythms across the cortex. Improved measurement of cortical rhythms will yield better conditions for establishing links between cortical activity and behavior, as well as bridging scales between the invasive intracranial measurements and noninvasive macroscale scalp measurements.
\end{abstract}

Keywords: electrocorticography, stereoelectroencephalography, spatial filters, neural oscillations, referencing

\section{Introduction}

Invasive, intracranial electroencephalography (iEEG) recordings from patients undergoing epilepsy monitoring have been tremendously valuable for examining neuronal activity. This is because iEEG provides both high temporal and spatial resolution that is impossible to achieve using solely noninvasive human neuroimaging (Engel et al., 2005; Jacobs and Kahana, 2010). Because of the superior spatial and temporal resolution of iEEG, combined with the possibility

*Corresponding author: nschaworonkow@ucsd.edu of simultaneous single-neuron recordings from humans (Suthana and Fried, 2012), these rare recordings provide a bridge between human cognition and decades of animal electrophysiology. The recordings display myriad types of complex activity, for instance prominent rhythms (Buzsáki et al., 2012) in several frequency bands, overlapping in time and space. Cortical rhythms have been examined during resting-state activity (Groppe et al., 2013; Frauscher et al., 2018) as well as during tasks (Jasper and Penfield, 1949; Halgren et al., 2019; Miller, 2019; Crone et al., 1998b). The rhythms show distinct spectral peaks, for instance in alpha- and beta-frequency range, 
distinct spatial distribution across rhythm types, for instance with beta-bursts prominent in the precentral gyrus, and the sensorimotor mu-rhythm in the postcentral gyrus (Stolk et al., 2019). Theta-rhythms are visible to a greater extent in invasive recordings, whereas in non-invasive recordings theta is mostly limited to mid frontal areas (Mitchell et al., 2008). Rhythms show distinct task-related modulation and intricate waveforms strongly deviating from sinusoids, with these non-sinusoidalities potentially providing improved physiological interpretability beyond oscillation power alone (Cole and Voytek, 2017).

For noninvasive electrophysiological recording techniques such as electroencephalography (EEG) and magnetoencephalography (MEG), source reconstruction techniques are commonly used to extract independent activity sources from sensor space data (Jas et al., 2018). This is often not the case for invasive electroencephalography (iEEG), where electrode-based approaches are dominant (Liu et al., 2015; Li et al., 2018; Arnulfo et al., 2015), which use a fixed way of referencing the data. The two most prevalent methods for referencing are to apply either a common average reference, with the aim to minimize common noise or distal activity, or to use bipolar reference, with the aim to extract locally generated signals. Source reconstruction for iEEG recordings has mostly employed in the context of localizing epileptic seizure focus. The main techniques used here are biophysical modeling (Pascarella et al., 2016; Dümpelmann et al., 2012; Fuchs et al., 2007; Chintaluri and Wójcik, 2015) and approaches using independent component analysis (Hindriks et al., 2018; Michelmann et al., 2018; Fahimi Hnazaee et al., 2020; Dümpelmann et al., 2012; Hu et al., 2007; Whitmer et al., 2010).

For examining high-frequency activity, an electrodebased approach (using a standard common average or bipolar reference) seems to be justified because of limited spatial spread of high-frequency signal content not exceeding inter-electrode distance (Dubey and Ray, 2019; Crone et al., 1998a), with sub-centimeter functional specificity (Flinker et al., 2011). In contrast to that, activity in lower frequency ranges displays an increased spatial spread, showing a high degree of correlation between neighboring electrode locations depending on oscillation frequency (Muller et al., 2016;
Crone et al., 1998b). Because of the spatial spread, it is expected that different rhythms contribute to activity of several electrodes due to spatial superposition. Therefore, multivariate separation techniques may improve measurement of cortical rhythms also in iEEG.

Here, we explore a data-driven spatial filtering method, spatio-spectral decomposition (SSD) for specifically extracting oscillatory sources in iEEG data. This technique, based on generalized eigenvalue decomposition, has been shown to be superior to for instance independent component analysis for extraction of oscillatory sources (Nikulin et al., 2011). Fig. 1 shows the underlying linear model of iEEG data and illustrates bipolar and common average referencing.

For recording iEEG data, a number of electrodes are placed on the cortical surface to acquire time series data. Each electrode picks up a mixture of signals from different types of cortical sources, determined by location and orientation of the generating sources and the biophysical properties of the tissue. This spatial mixing is given by the forward model and is assumed to be linear here (Parra et al., 2005). The SSD approach helps in recovering distinct neuronal sources from the activity recorded via the electrodes, i.e., it estimates a backward model in the form of spatial filters, in order to best measure the temporal dynamics of oscillations and their associated features of interest. Data referencing can be viewed as the application of a spatial filter, in which the trace of each electrode is multiplied with a specific weight. For instance, in the case of a bipolar filter, the spatial filter is a vector with as many entries as electrodes, containing weights -1 and +1 for two selected electrodes and zero for all other electrodes. For each time point, the dot product of the electrode data with the spatial filter is taken to yield the corresponding entry for the component trace. The spatial filter vector is the same for all time points and this operation can be performed efficiently by matrix multiplication.

It is important to make a distinction between spatial filters and the spatial patterns associated with each filter. A spatial filter assigns a weight to each electrode that quantifies how much each electrode contributes to the extracted component. A spatial filter is generally not interpretable (Haufe et al., 2014b), however, 
in the sense that the magnitude of the weights directly reflects the contribution of the source to the spatially filtered signal. This information about spatial origin of a component can be found in the spatial pattern, which can be computed for each spatial filter and reflects the mapping of sources onto measured electrode signals, showing the strength and polarity of a source signal on all electrodes. The spatial patterns are computed by matrix inversion of the spatial filters. It can be seen that although the spatial filters in Fig. 1 have different structure respectively, the associated spatial patterns are quite similar, reflecting a source originating in the sensorimotor region.

In this article, we use spatial filters to investigate rhythms present in mainly the alpha and beta-frequency bands in human iEEG recordings. We illustrate that the activity spread of individual rhythms can exceed inter-electrode distance and show that spatial mixing of rhythms in intracranial recordings can affect the oscillatory power of a given rhythm and alter its non-sinusoidal waveform shape when sources are mixed. We demonstrate how spatial filtering can identify rhythms that otherwise may not be apparent in the data due to masking by other stronger oscillatory contributions, from low signal-to-noise ratio (SNR), and/or from destructive interference. We also extract dominant rhythms in a resting state dataset with spatial filters and discuss variability across participants in presence of detected rhythms. Additionally, we illustrate how spatial filtering can be used as a powerful way to remove band-limited noise, without artefacts from temporal bandstop-filtering. While the employed spatial filtering methods are already used in analysis of noninvasive recordings, the aim here is to also highlight the specific benefits of using datadriven spatial filters for invasive electrophysiological recordings. Improved measurement of rhythms will aid bridging the scales from recordings obtained invasively to noninvasive recording techniques.

\section{Materials and Methods}

\subsection{Experimental recordings}

We analyzed openly available datasets from a library of intracranial recordings (Miller, 2019). We primar- ily used the dataset fixation_pwrlaw where participants fixated on a target location for several minutes, as our focus here is physiological rhythms in the resting state. For the single participant spatial mixing illustration in Fig. 2 as well as Fig. S1 and to show that spatial filtering preserves oscillatory task dynamics in Fig. S2 we used the dataset motor_basic. In addition, we used one recording from the faces_basicdataset to demonstrate the application of spatial filters for strip electrode recordings for several leads that are in close vicinity and one recording for removing noise with a specific spectral profile. We include the required ethics statement for each of those datasets in the following, as mandated by the data usage requirements of the data library.

\subsubsection{Ethics statements}

Data set fixation_pwrlaw: Ethics statement: All patients participated in a purely voluntary manner, after providing informed written consent, under experimental protocols approved by the Institutional Review Board of the University of Washington (\#12193). All patient data was anonymized according to IRB protocol, in accordance with HIPAA mandate. These data originally appeared in the manuscript "PowerLaw Scaling in the Brain Surface Electric Potential" published in PLoS Computational Biology in 2009 (Miller et al., 2009).

Dataset motor_basic: Participants in this dataset performed hand or tongue movements with timing based on a cue, with movement contralateral to placement of the recording grid. Cues were presented as written words in a $10 \times 10 \mathrm{~cm}$ presentation window, within a distance of $0.75-1 \mathrm{~m}$ from participants. The analyzed dataset features a 39 year old female participant with a 5 x 5 electrode array, with an inter-electrode spacing of $10 \mathrm{~mm}$, with a $4 \mathrm{~mm}$ diameter of each electrode. The sampling frequency was $1000 \mathrm{~Hz}$, acquired with the sample recording system as above and hardware band-pass filtered in the same range.

Data set faces_basic: Ethics statement: All patients participated in a purely voluntary manner, after providing informed written consent, under experimental protocols approved by the Institutional Review Board of the University of Washington (\#12193). All patient 
bioRxiv preprint doi: https://doi.org/10.1101/2021.02.26.433127; this version posted February 28, 2021. The copyright holder for this preprint (which was not certified by peer review) is the author/funder, who has granted bioRxiv a license to display the preprint in perpetuity. It is made available under aCC-BY-NC-ND 4.0 International license.

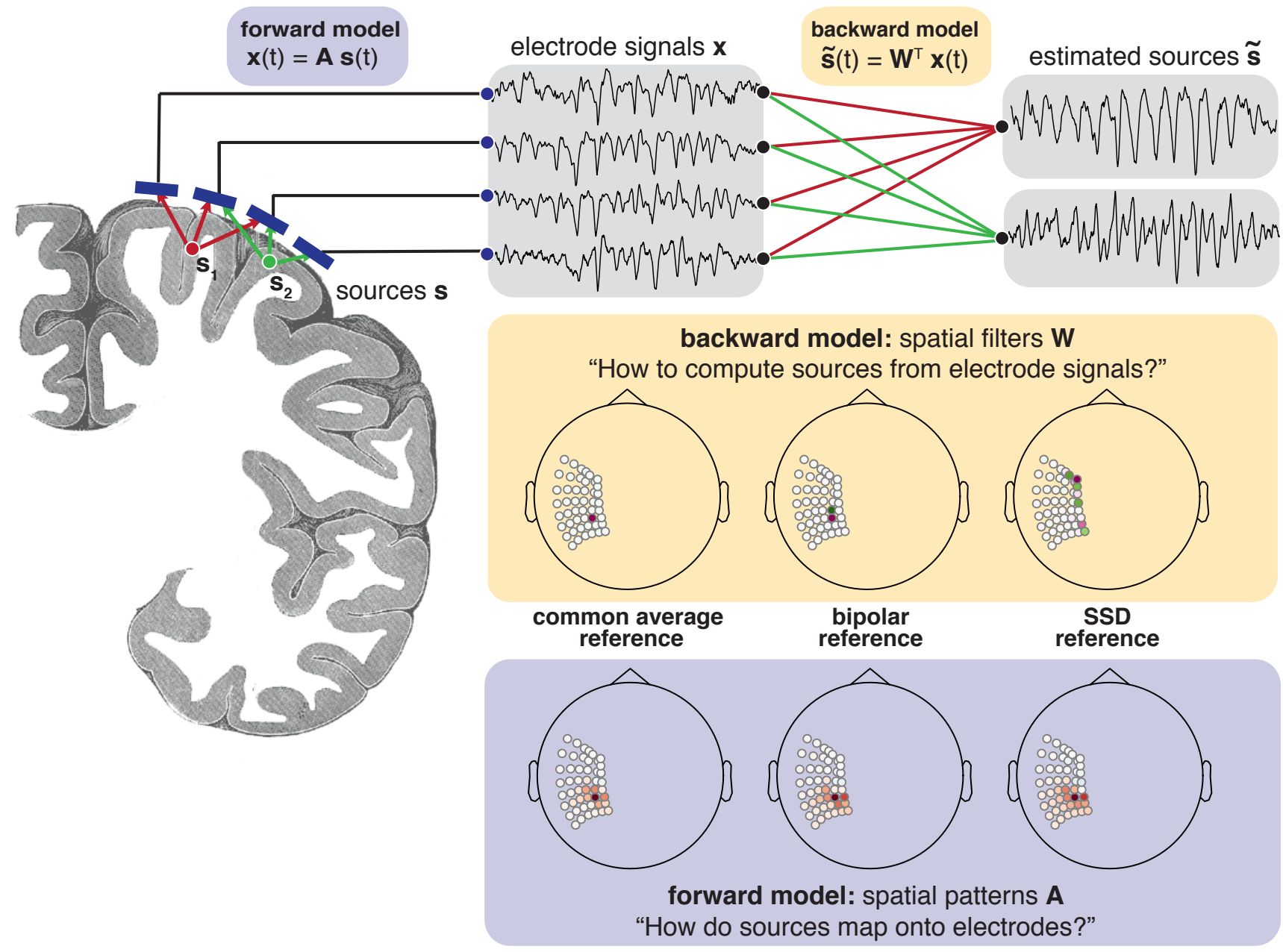

Figure 1: Generative linear model of electrophysiological data. Sources $s$ in the gray matter mix according to the forward model $A$ with the corresponding propagation of currents through tissue to the electrodes. The resulting signals $x$ are recorded with electrodes placed on the cortical surface. The objective is to estimate the sources $\tilde{s}$ from the electrode signals $x$ with a backward model $W$. Three different backward models are illustrated with one specific example of their respective corresponding spatial filters and patterns. While the spatial filters can look quite different from each other, the spatial patterns point to a similar spatial origin of the extracted signal. Image source for coronal cut: public domain Gray's anatomy plate 718 (Gray, 1918).

data was anonymized according to IRB protocol, in accordance with HIPAA mandate. These data originally appeared in the manuscript "Spontaneous Decoding of the Timing and Content of Human Object Perception from Cortical Surface Recordings Reveals Complementary Information in the Event-Related Potential and Broadband Spectral Change" published in PLoS Computational Biology in 2016 (Miller et al., 2016).

\subsubsection{Participants}

For resting state group analyses, the data from 20 participants was used. The mean age was $31.1 \pm 9.5$ (mean \pm standard deviation), 9 female, 7 male. For four participants, age and gender information was not available.

\subsubsection{Experimental design and recording setup}

Dataset fixation_pwrlaw:The task was a fixation task where participants fixated on a fixation cross placed on the wall in three meters distance for several minutes (mean data length: $157 \pm 51$ s). Intracranial recordings were made from subdural electrode arrays (mean number of electrodes: $60 \pm 12$ ), with an inter-electrode spacing of $10 \mathrm{~mm}$, with a $4 \mathrm{~mm}$ diameter of each electrode. For most participants, data was available with a sample rate of $1000 \mathrm{~Hz}$ and was resampled to $1000 \mathrm{~Hz}$ for participants with a higher sam- 
pling rate. The recordings were made with Neuroscan Synamps ${ }^{2}$ amplifiers (Compumedics-Neuroscan, San Antonio, TX) in conjunction with a clinical recording system (XLTEK or Nicolet-BMSI). A common ground and reference electrode, placed on the scalp, was used. A hardware band-pass filter from $0.15 \mathrm{~Hz}$ to $200 \mathrm{~Hz}$ was applied.

Dataset motor_basic: Participants in this dataset performed hand or tongue movements with timing based on a cue, with movement contralateral to placement of the recording grid. Cues were presented as written words in a $10 \times 10 \mathrm{~cm}$ presentation window, within a distance of $0.75-1 \mathrm{~m}$ from participants. The analyzed dataset features a 39 year old female participant with a 5 x 5 electrode array, with an inter-electrode spacing of $10 \mathrm{~mm}$, with a $4 \mathrm{~mm}$ diameter of each electrode. The sampling frequency was $1000 \mathrm{~Hz}$, acquired with the sample recording system as above and hardware band-pass filtered in the same range.

Dataset faces_basic: Participants in this dataset performed a simple visual discrimination task. The electrodes had $4 \mathrm{~mm}$ diameter and $10 \mathrm{~mm}$ inter-electrode spacing, with silastic embedding. We selected three electrode leads over the left parietal hemisphere for analysis. The sampling frequency was $1000 \mathrm{~Hz}$, acquired with the sample recording system as above and hardware band-pass filtered in the same range, the signals were measured with respect to a scalp reference and ground.

\subsection{Data analysis}

Data analysis was performed using Python in conjunction with MNE v.0.20.4 (Gramfort et al., 2013). Analysis code necessary to produce the figures in the manuscript from raw data is available at: https://github.com/nschawor/ieeg-spatial-filters-ssd

\subsubsection{Spectral analysis and parametrization}

Power spectra were calculated with Welch's method (3 s window length, $0 \%$ overlap). The spectral parameterization method and toolbox of (Donoghue et al., 2020) (version 1.0.0) was employed for determination of peak frequencies. In this method, the power spectrum is modeled as a superposition of aperiodic and oscillatory components, which allows to distinguish between oscillatory and aperiodic contributions to the power spectrum. The power spectrum $P(f)$ for each frequency $f$ is expressed as:

$$
P(f)=L(f)+\sum_{n} G_{n}(f) .
$$

With the aperiodic contribution $L(f)$ expressed as:

$$
L(f)=b-\log \left[f^{\chi}\right],
$$

with a constant offset $b$ and the aperiodic exponent $\chi$. When the power spectrum is plotted on a log-log axis, the aperiodic exponent $\chi$ corresponds to the slope of a line. Each oscillatory contribution $G_{n}(f)$ is modelled as a Gaussian peak:

$$
G_{n}(f)=a_{n} \exp \left[-\frac{\left(f-\mu_{n}\right)^{2}}{2 \sigma_{n}^{2}}\right],
$$

with $a_{n}$ as the amplitude, $\mu_{n}$ as the peak frequency and $\sigma_{n}$ as the bandwidth of each component. The number of oscillatory components is determined from the data, with the option to set a maximum number of components as a parameter. The model assumption is that oscillatory and aperiodic processes are separable. Settings for the spectral parameterization algorithm were: peak width limits: $(0.5,12.0)$; maximum number of peaks: 5 ; minimum peak amplitude exceeding the aperiodic fit: 0.0; peak threshold: 2.0 ; and aperiodic mode: 'fixed'. Here, we only extracted the peak frequencies and bandwidths for each electrode of each participant, discarding the aperiodic exponent.

\subsubsection{Calculation of spatial filters}

We estimate spatial filters via spatio-spectral decomposition (SSD) (Nikulin et al., 2011), which specifically maximizes spectral power in a frequency band of interest, while minimizing spectral power in flanking frequency bands. This procedure enhances the height of spectral peaks over the 1/f-contribution, exploiting specifically the typical narrowband peak structure of neural oscillations. The underlying data model for the method assumes that the measured time series $\mathbf{X}$ (a matrix with $t$ samples and $k$ electrodes) constitute a linear superposition of signal $\mathbf{X}_{\mathrm{S}}$ and noise $\mathbf{X}_{\mathbf{N}}$ contributions in the data. In the particular case, signal here means oscillations in a narrow frequency band, 
while noise represents the signal in the neighboring frequency bands. The estimation procedure uses temporally band-pass filtered activity, centered on a peak frequency with a specified bandwidth. The choice of peak frequency and bandwidth was informed by spectral parametrization of signals from all electrodes. Following the original paper (Nikulin et al., 2011), we use a $4^{\text {th }}$ order Butterworth filter for temporally filtering the respective signal and noise contributions. The covariance matrices of the signal and noise contributions are calculated on the basis of the band-pass filtered electrode activity.

$$
\mathbf{X}=\mathbf{X}_{S}+\mathbf{X}_{N}
$$

signal covariance $\mathbf{C}_{\mathbf{S}}=\mathbf{X}_{\mathbf{S}}{ }^{T} \mathbf{X}_{\mathbf{S}}$ with $\mathbf{C}_{\mathbf{S}} \in \mathbb{R}^{k \times k}$ noise covariance $\mathbf{C}_{\mathbf{N}}=\mathbf{X}_{\mathbf{N}}^{T} \mathbf{X}_{\mathbf{N}}$ with $\mathbf{C}_{\mathbf{N}} \in \mathbb{R}^{k \times k}$

The objective is to find a spatial filter $\mathbf{w}$, which maximizes the power of the projected signal $P_{S}$, while minimizing the power of the projected noise $P_{N}$.

$$
\operatorname{SNR}(\mathbf{w})=\frac{P_{S}}{P_{N}}=\frac{\operatorname{var}\left(\mathbf{w}^{T} \mathbf{X}_{S}\right)}{\operatorname{var}\left(\mathbf{w}^{T} \mathbf{X}_{N}\right)}=\frac{\mathbf{w}^{T} \mathbf{C}_{\mathbf{S}} \mathbf{w}}{\mathbf{w}^{T} \mathbf{C}_{\mathbf{N}} \mathbf{w}}
$$

This Rayleigh quotient can be transformed into a generalized eigenvalue problem, which allows efficient and fast computation. In matrix form, the above equation can be written as:

$$
\mathrm{C}_{\mathrm{S}} \mathbf{W}=\mathrm{C}_{\mathbf{N}} \mathbf{W} \Lambda
$$

where $\mathrm{W}$ is the matrix of all spatial filters, and $\Lambda$ is the unity matrix with the corresponding eigenvalues on the diagonal. While the spatial filters are estimated with the aid of covariance matrices obtained from narrowband activity, the spatial filters are then applied on the broadband signal. The application to a broadband signal ensures that features of activity originating from the same spatial location will also be extracted by the spatial filter, for instance the harmonics of a non-sinusoidal signal. The number of components returned by SSD is equal to the number of electrodes, with the components ordered by relative SNR in the frequency band of interest.

The peak frequency of estimated SSD components can differ slightly from the target peak frequency used to define the signal contribution. Therefore, after spatial filter estimation, the peak frequency and the SNR of each component (spectral peak height exceeding the $1 /$ f-contribution) was assessed by calculating the power spectra and parametrization of them with the same parameter settings as for the electrode signals. Components exceeding a SNR-value of $5 \mathrm{~dB}$ were retained. This will discard weak rhythms, but the main objective here is to identify rhythms using a common threshold in order to make comparisons across participants. This was also done to illustrate a caveat in iEEG analyses, which commonly involves pooling of electrodes across participants, i.e., underlying here is the assumption of similar SNR across participants. The value of the SNR threshold was chosen in accordance with our previous studies (Schaworonkow et al., 2019), which were set to examine temporally resolved features of oscillations, e.g., instantaneous phase.

\subsubsection{Calculation of spatial patterns}

Spatial patterns for interpretation of the spatial origin of the extracted component are obtained with aid of covariance matrices calculated for the signal component in the frequency band of interest and the pseudoinverse of the spatial filters (Haufe et al., 2014b). To illustrate spatial spread of oscillatory components, we analysed the topography of spatial pattern coefficients. For each component, the absolute value of the associated spatial pattern coefficients was taken and the values were then divided by the maximum value. The maximum spatial pattern coefficient in a distance of $2.5 \mathrm{~cm}$ around the maximum (distance value determined by Euclidean distance) was extracted to assess contribution of a single component onto several electrode signals.

\subsubsection{Waveform shape analysis}

The bycycle toolbox (Cole and Voytek, 2019) was used for detecting and quantifying burst features in the time domain, using the following steps: First, a narrow band-pass filter (finite impulse response filter, peak frequency $\pm 3 \mathrm{~Hz}$ ) was used for identification of zero-crossings. With aid of zero-crossings, cycle features are determined on broadband filtered $(1-45 \mathrm{~Hz})$ data. All cycles that pass predefined criteria were classified as bursts. We used the following parameter settings for determining bursts, consistent 
across datasets: minimum of three present cycles, amplitude fraction threshold $=0.75$, amplitude consistency threshold: 0.5 , period consistency threshold: 0.5 , monotonicity threshold: 0.5 . An amplitude fraction threshold of 0.75 retains only the cycles exceeding an amplitude higher than the $75^{\text {th }}$ percentile. The relatively high threshold was chosen to allow for improved measurement of asymmetries, as burst occurrence may be quite infrequent. Then, mean waveform features across burst cycles (e.g., voltage amplitude and cycle frequency) were calculated for each component. A main focus here was the measurement of waveform shape asymmetries, i.e., peak-trough asymmetry, where the fraction spent in peak time (time from rising flank zero-crossing to falling flank zerocrossing) differs from the fraction spent in trough time (time from falling flank zero-crossing to rising flank zero-crossing), as well as rise-decay asymmetry, where the time taken from peak to trough differs from the time taken from trough to peak.

\subsubsection{Noise removal with spatial filters}

For removing noise with a specific spectral profile, we estimate spatial filters for maximizing SNR around the frequency peak that should be removed, e.g., $60 \mathrm{~Hz}$ for line noise. Then these components are subtracted from the raw signal with a linear operation:

$$
\mathbf{X}_{\text {cleaned }}=\mathbf{X}-\sum_{j=1}^{N} \mathbf{a}_{j} \mathbf{s}_{j}
$$

with $\mathbf{X}$ the raw signal matrix, $N$ the number of components to remove, $\mathbf{a}_{j}$ the spatial pattern associated with component $j$ and $\mathbf{s}_{j}$ the time course of the SSD component $j . N$ can be determined by inspection of the power spectra of the estimated components, and removing components iteratively until the noise level reaches a sufficiently low state.

\section{Results}

\subsection{Several rhythms contribute to intracranial activ- ity from single electrodes}

First, we illustrate how activity taken from single intracranial electrodes shows a mixture of several different rhythms. Each electrode features sensorimotor bursts in the alpha-frequency range, as indicated by a peak around $10 \mathrm{~Hz}$ in the spectral domain (Fig. 2A) and cycles with a period of approximately $100 \mathrm{~ms}$ in the time domain (Fig. 2B). We compute data-driven spatial filters using narrowband activity in the alphafrequency range defined as the signal contribution and flanking frequency bands defined as the noise contribution. The estimated spatial filters are then applied on broadband activity. The spectra and examples of the time domain activity of the three components with highest SNR are shown in Fig. 2D and Fig. 2E. The components display an increase in relative SNR (peak amplitude height over 1/f-contribution), compared to the raw electrode signals. The ordering of the components is according to the SNR in alpha-range, with the strongest relative SNR rhythm shown first. The SNR for all components can be seen in Fig. 2F showing that SNR is highest for the first component, and a fast drop off in SNR. This ordering enables fast inspection, as only the first couple of components typically contain activity in the frequency band of interest with sufficient SNR.

Of note in the example is that the first component is of much smaller total power than the second and third components, as can be seen in the power spectrum. The large relative SNR of the oscillation stems in part from the low power of the $1 / \mathrm{f}$-contribution. In electrode 1 and 3 , this component has a large contribution, as evidenced by large coefficients of the spatial pattern. But due to contributions of other components with a higher overall amplitude, this component is obscured on the level of electrodes. Only the activity in the alpha frequency band and surrounding bands is used to estimate spatial filters, but since harmonics originate from the same spatial location, due to the non-sinusoidal nature of the oscillation, the application of the spatial filters retains the SNR in the harmonic bands when the spatial filter is applied on a broadband signal.

Examining the spatial patterns associated with each component (Fig. 2I), it is evident that the multiple rhythms co-occur in a small area, which results in each component contributing to the activity of the electrodes as indicated by large coefficients in the spatial pattern. In the case of tangentially orientated sources, the sign of the contribution can switch be- 
tween neighboring electrodes. Depending on the spatial mixing of those rhythms, they can cancel out or enhance each other during specific time periods, due to changes in their phase relationship. In time periods where components of comparable amplitude are phase-aligned, constructive interference takes place, resulting in a large amplitude of the electrode signal. In periods with a phase shift reaching $\pi$ or 180 degrees, with peaks of one component coinciding with troughs of another, destructive interference can result in a low electrode signal amplitude, even though oscillations are still present in the individual components (see time points marked with red box in Fig. 2B). Therefore, by constructive and destructive interference, changes in the power of the electrode signal can reflect changes in synchronization of rhythms across space (Schaworonkow and Nikulin, 2019), and do not necessarily reflect changes in the oscillation strength of the source signals. Disentangling these different possible causes of changes in oscillatory power cannot be done based solely on activity that displays a large degree of spatial mixing and data-driven spatial filters may be helpful to distinguish such phenomena.

Examining the spatial filters for each component (Fig. 2H) they resemble but also diverge from bipolar or Laplaciantype spatial filters. The spatial filter associated with component 1 has a bipolar-type form, but with the advantage that the direction along which the bipolar derivation is taken is learned from data. In Fig. S1 we show the same traces for common average referenced electrodes, which shows higher SNR compared to the common reference, but still exhibits spatial mixing, as well as bipolar derivations in two directions (anterior to posterior and lateral to medial). Additionally, we show in Fig. S2 that task-related temporal dynamics are preserved by SSD spatial filtering, in line with other referencing methods, showing high consistency. The main argument here is not that SSD will achieve the highest SNR, but that referencing to capture specific sources is dependent on properties of the source and that not all sources will be captured best by a fixed referencing scheme, which utility may depend on the local cortex morphology. In that sense, using data-driven spatial filters uses information given by the multivariate structure of recordings to a greater extent.

\subsection{Improvement of signal to noise ratio for $s E E G$ signals}

There are many ways to reference stereoelectroencephalography (sEEG) recordings, ranging from monopolar, bipolar, common average or Laplacian referencing (Li et al., 2018). The choice of reference is a researcher degree of freedom. Fig. 3 shows application of SSD for a recording consisting of three close-by sEEG leads, as shown in Fig. 3A. Fig. 3B shows time domain examples of electrode signals that were first common average referenced within a lead as well as examples for bipolar referencing (choosing a neighboring electrode on the same lead as the 2nd electrode), compared to the first SSD component for a peak frequency that was selected according to the spectrum of electrode signals, as is visible in Fig. 3C for all three types of signals. It can be seen that SNR of rhythms of interest was improved in sEEG recordings by spatial filtering using SSD. In the case of the $8.2 \mathrm{~Hz}$ component, an enhancement of the spectral peak for the harmonic frequency is also visible, demonstrating the potential for SSD for isolating non-sinusoidal rhythms. Additionally, all ) signals were submitted simultaneously to the SSD procedure, without subselection, making it possible to combine information from multiple leads and electrode configurations efficiently. Using SSD results in less bias due to fixed reference choice, as learning the filter coefficients from the data enables recovery of rhythms in a flexible way regardless of their location and dipole orientation.

\subsection{Determining number and spatial extent of rhythms}

Having demonstrated basic properties of spatial filtering with SSD, we turn to a number of physiological aspects to consider. Fig. 4B shows time domain examples of two closely neighboring electrodes (black traces) with oscillatory activity in the same frequency band. The time domain activity of these electrodes looks similar, with prominent alpha-band oscillations. While these two electrodes look similar, it is unclear whether this is due to several independent rhythms with the same peak frequency, or one underlying source that is projecting onto both electrodes. Estimating spatial filters with SSD shows the existence of two components with alpha-rhythm activity, one 
A

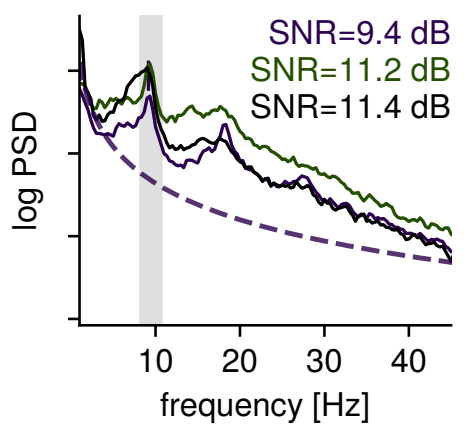

D

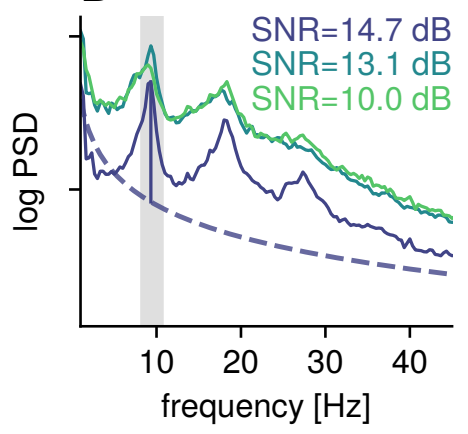

$\mathrm{SNR}=14.7 \mathrm{~dB}$

$S N R=13.1 \mathrm{~dB}$

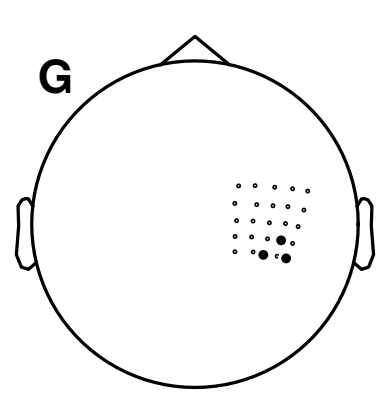

B

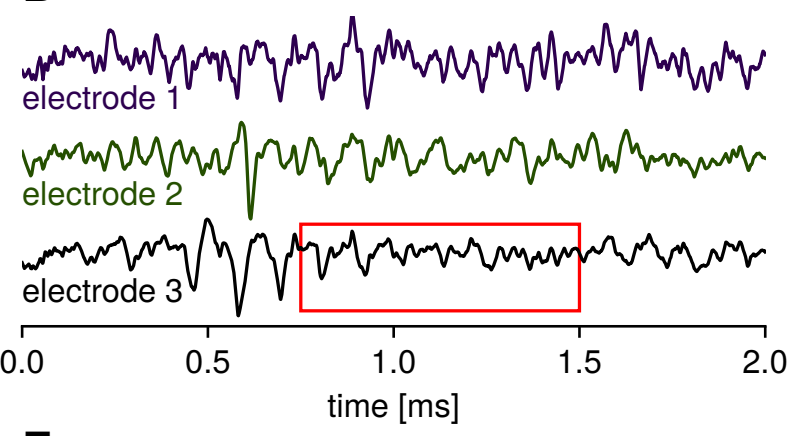

E

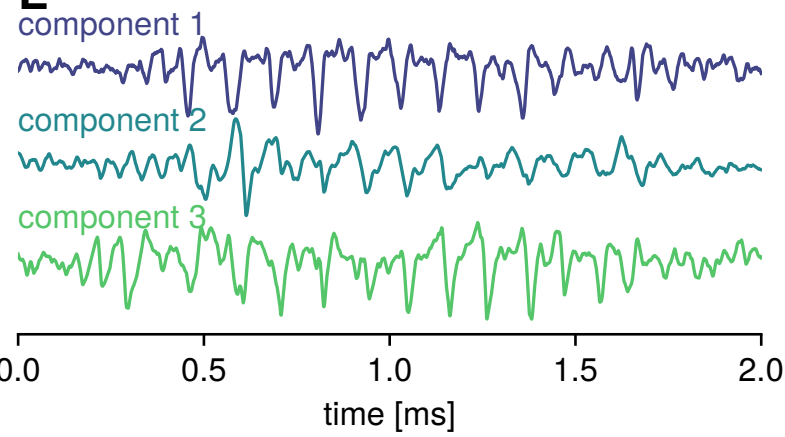

C
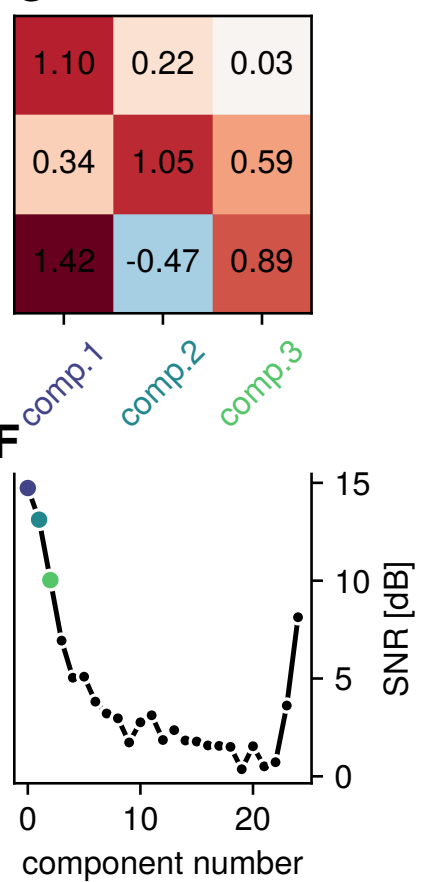

H
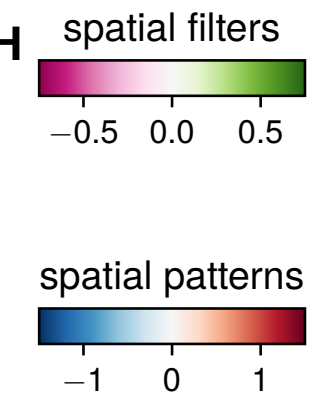

00000

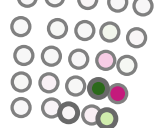

O 000

component 1

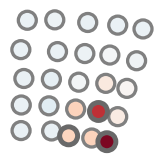

00000

00000

00000

O 000

component 2

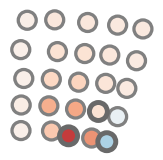

00000

00000

00000

O 000

o 000

component 3

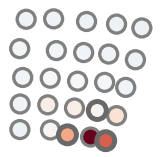

Figure 2: Example: spatial mixing of sensorimotor rhythms for one participant. A) Power spectral densities for three channels along the sensorimotor strip. The gray bar indicates the frequency band defined as the signal contributoon for estimating the SSD spatial filters. The power spectrum shows a peak frequency in the alpha-band, with additional harmonic peaks. The channels were selected a according to highest SNR in the chosen frequency range. B) The corresponding signal in the time domain showing oscillatory bursts in the alpha-band, amplitudes are normalized for comparison of time courses. The red box marks a time period in which less pronounced oscillations can be seen in the electrode signals, but the oscillatory power of the constituent SSD components is not decreased. C) Coefficients in the spatial patterns for the selected electrodes, i.e., electrode 2 can be approximated as a linear combination of: $e_{2}=0.34$ component $_{1}+1.05$ component $_{2}+0.59$ component $_{3}$. D) Power spectral densities for the first three components as estimated by SSD, showing a higher alpha-SNR, with less spectral peaks in flanking frequency bands. E) Time domain signal for the corresponding three components, showing pronounced sensorimotor bursts, normalized amplitudes for comparison of time courses. F) SNR per component, for all 25 components. The SNR drops off fast, only a number of components need to be inspected. For the components last in the sequence, the SNR increases as rhythms in flanking bands increase spectral power also in the band of interest. G) Approximate location of the ECoG-grid in head coordinates. The black markers highlight the electrodes shown in A) and B). H) Spatial filter coefficients showing similarity to bipolar and Laplacian-type filters. I) Spatial pattern coefficients showing focal contributions from sources along the sensorimotor strip.

strong alpha-rhythm (in terms of relative SNR) and a second alpha-rhythm with much smaller spectral power (Fig. 4C), which would be masked in individual electrode traces. Of note is that the spatial spread of these rhythms exceeds inter-electrode spac- ing, with the same rhythm having large contributions to the activity of neighboring channels, as indicated by the spatial pattern coefficients showing high values for a cluster of electrodes. The components are closely overlapping in space as can be seen from the 
A

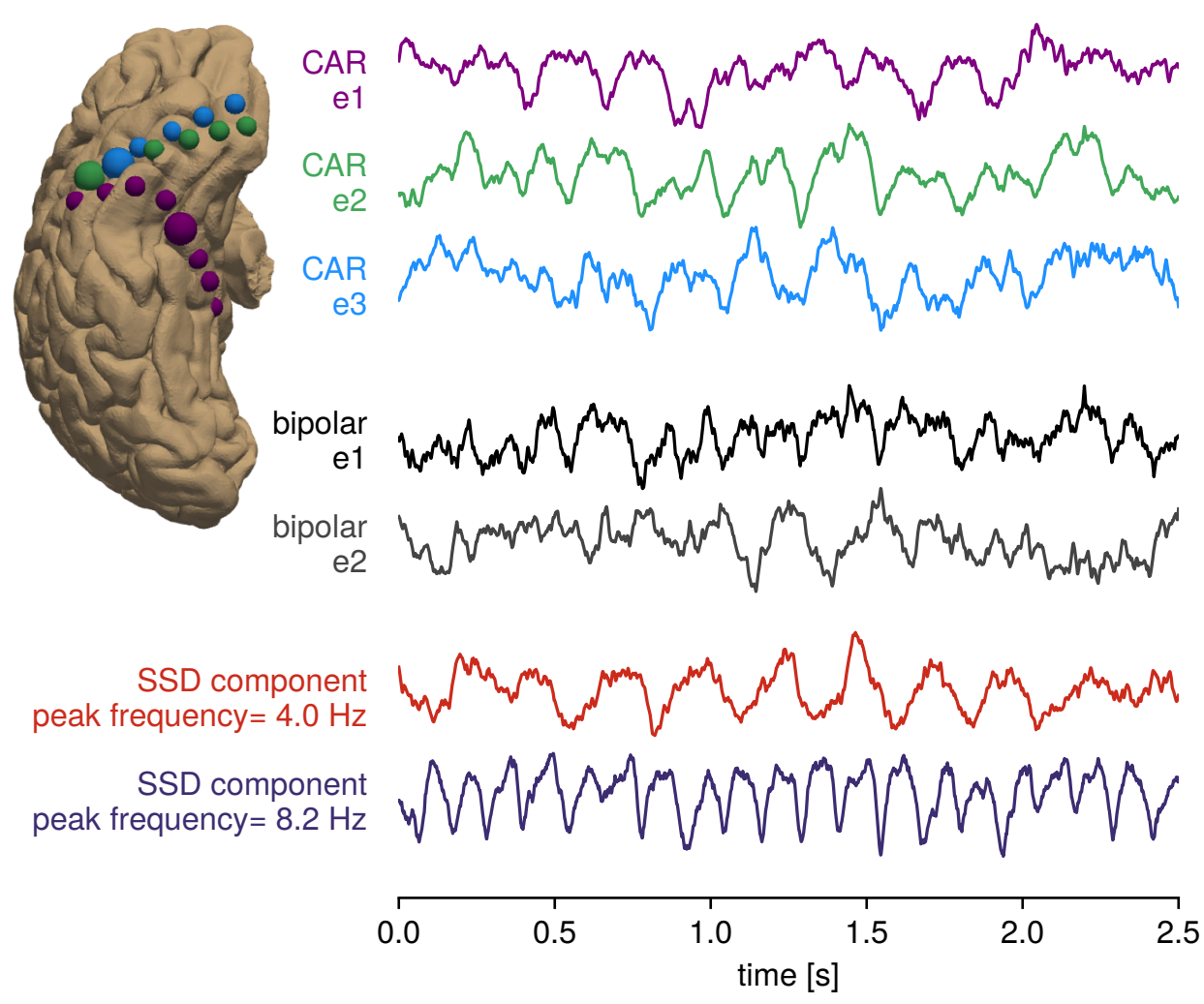

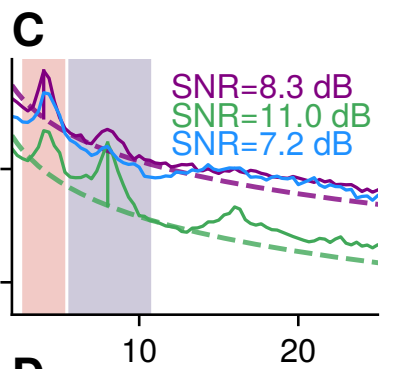
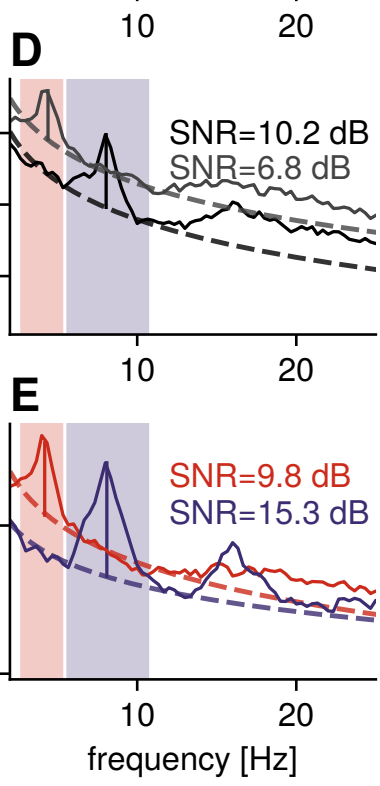

Figure 3: Illustration: Increase of relative SNR for sEEG A) Three sEEG leads (blue, green, purple color respectively) plotted on cortical surface, ventral view. The electrodes highlighted with a larger circle size correspond to the colored traces. B) Time domain signals for common average referenced (CAR) signals, bipolar referenced signals and strongest SSD components for two selected peak frequencies, with bandwidth highlighted with colored boxes in spectral plots. C) Power spectral densities for common average referenced signals, showing multiple peak frequencies in the spectrum. D) Power spectral densities for bipolar-referenced signals. E) Power spectral densities for SSD components, showing an increased SNR over standard referencing.

topography of spatial patterns (Fig. 4A). By applying data-driven spatial filters, the rhythms close in frequency and space can be disentangled, enhancing the detectability of small amplitude rhythms, for instance.

To go beyond a single participant, we analyzed the coefficients of spatial patterns for our resting dataset. Fig. 4D shows the spatial spread of different rhythms, as measured by the spatial pattern coefficients of electrodes neighboring the electrode with the maximum coefficient. It is visible that rhythms can have large contributions onto several electrodes, as indicated by neighboring channels with high coefficients. We show examples of rhythms with high spatial spread in Fig. 4E, top row. A key point we want to highlight is that the spatial spread can also be small (see Fig. 4E, bottom row), with only one singular maximum for one electrode relative to other electrodes. In such a case, a standard common average reference might be a sufficient but more simple approach for investigating rhythms. However, in the case of a rhythm of a large spatial spread across a large number of electrodes however, this rhythm may be attenuated when using a common average reference. Thus, the benefit of using data-driven spatial filters is that they may work in both cases. In addition, using spatial patterns as estimated from the covariance matrix between channels may be helpful as a tool for data exploration to evaluate this factor, because the spatial correlations across electrodes for different present rhythms cannot be known a priori.

In general, signal decomposition techniques like SSD can be used for dimensionality reduction, keeping 
only the $N$ components that contribute most strongly to the signal in the band of interest, and projecting out all other components to limit analyses to a specific subspace. The determination of which components to keep can be made using a threshold criterion based on relative SNR (Nikulin et al., 2011; Haufe et al., $2014 \mathrm{a}$ ), with the aid of a bootstrapping procedure (Zuure et al., 2020), or based on physiological considerations such as focusing on rhythms originating from a specific location, which can be determined with aid of the spatial patterns.

\subsection{Variability of resting rhythms across the cortex}

To demonstrate how data-driven spatial filters can be used for data exploration, we assess the resting rhythms in the frequency range of 5 to $20 \mathrm{~Hz}$ for different participants in an iEEG resting state dataset. For this analysis, spatial filters were computed separately for specific frequency bands, with the frequency ranges selected via spectral parameterization of electrode signal to identify putative oscillations that exhibit narrowband power above the 1/f-contribution. The components with SNR exceeding a specified threshold were retained. Fig. 5A shows the spatial distribution of different rhythms extracted with SSD for individual participants. The location of the respective component marker reflects the electrode location of the maximum spatial pattern coefficient. In general, there are several rhythms detectable in the 5 to $20 \mathrm{~Hz}$ range. But there is considerable variation in peak frequency and measured SNR across participants, as indicated by a variety of possible component arrangements across the cortex.

We also show the peak frequency of all identified components in Fig. 5B and C. We find a distribution similar to Groppe et al. (2013), where there are more rhythms detected with a peak frequency around 7 as well as $16 \mathrm{~Hz}$, and fewer rhythms with a peak frequency around $10 \mathrm{~Hz}$, in contrast to non-invasive electrophysiological measurements. This distribution of peak frequencies is possibly related to the spatial bias of electrode placement, wherein most are placed over sensoriomotor and temporal areas, with less coverage over occipital areas, as determined by clinical needs. Additionally, the recording spans the duration of several minutes, during which time the partici- pant's behavior is relatively unconstrained and variable. However, such a duration is typical for restingstate or baseline recordings across different studies. Another factor to consider, especially in iEEG data, is the fact that patients have the grids implanted for clinical reasons, with different pathologies and different medication status, which might contribute to the observed variability. Nevertheless, variability in peak frequencies and oscillatory SNR is also observed in non-invasive electrophysiological measurements. For iEEG, this might be more of a concern, since a smaller number of participants are usually included per study, compared to studies using non-invasive measurements.

The key point we aim to illustrate here is that rhythms are present with different SNR and variation in peak frequency across participants, and canonical oscillations of interest may or may not be detectable in individual participants in iEEG data, given the large between participant variability. The variability may contribute to inconsistent results, as temporal bandpass filtering of activity in a certain area within a predefined frequency band might not actually reflect oscillatory dynamics, but might capture only contributions from 1/f-components. Applying data-driven spatial filters can aid in verifying the presence and spatial origin of rhythms of interest, while also improving measurements of their precise temporal dynamics by increasing the SNR.

\subsection{Waveform shape and spatial mixing}

Neuronal oscillations are often of a non-sinusoidal shape, for instance in the form of a pronounced arcshape in the case of the sensorimotor mu-rhythm (e.g., see Fig. 2, component 1). While waveform can be informative about neuronal processing (Cole and Voytek, 2017), the detectability of waveform shape requires a high enough SNR to capture harmonic frequencies, which may not be detectable with a high level of $1 / \mathrm{f}$-noise. While non-sinusoidality is also present in noninvasive signals, the difficulty there is that it is often obscured by spatial mixing and a low SNR. Therefore, invasive cortical recordings provide an excellent opportunity to study waveform shape. As the amplitude envelope of rhythms in the same frequency band tends to co-fluctuate (Hipp et al., 2012) 
A

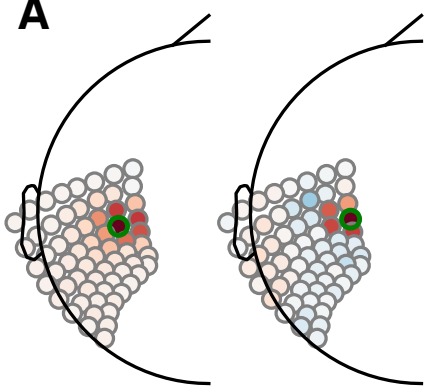

B

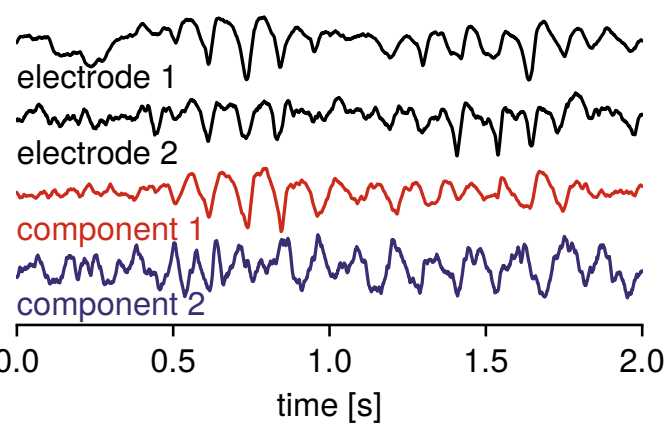

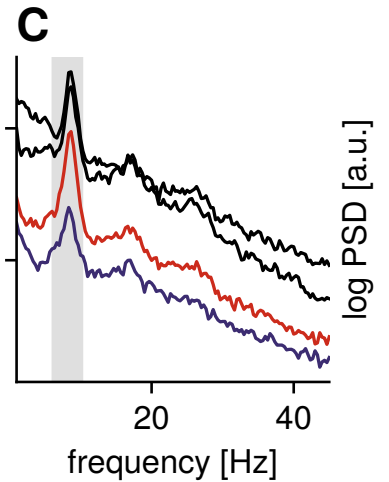

frequency $[\mathrm{Hz}]$

E spatial spread over multiple electrodes
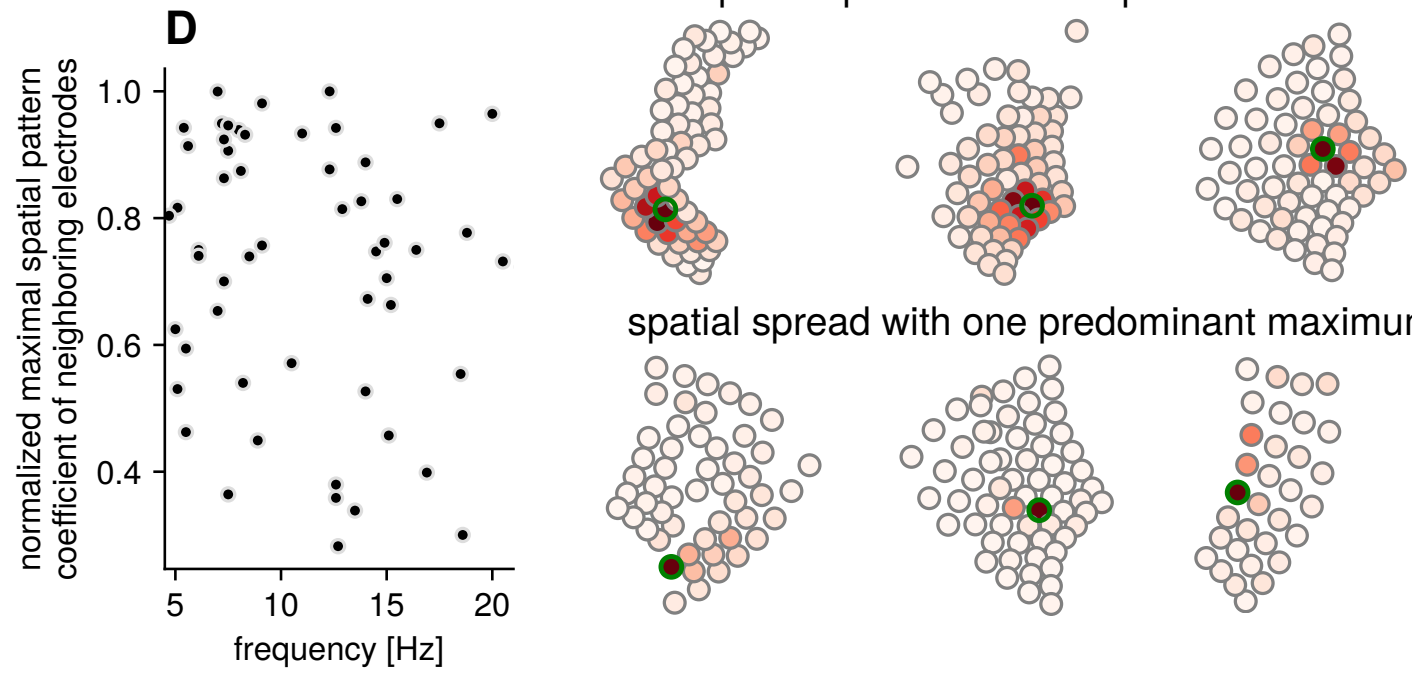

spatial spread with one predominant maximum
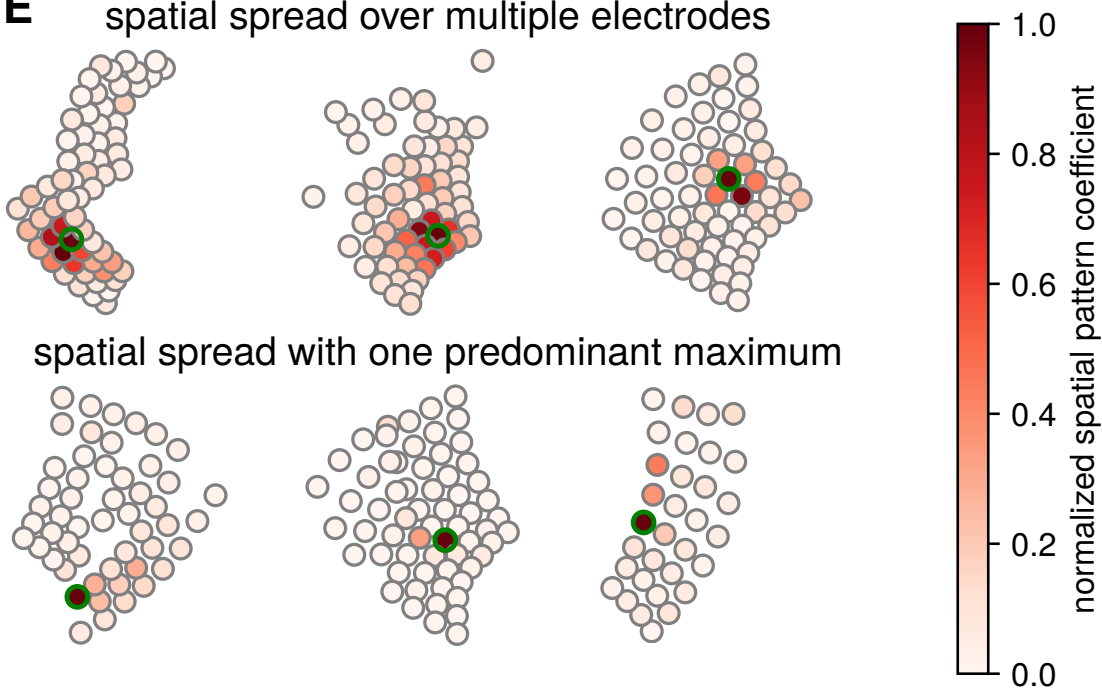

Figure 4: Identifying independent sources A) Spatial patterns for two components with electrodes highlighted in green. B) Time domain activity for two neighboring electrodes (black) and the top SNR components for alpha range (gray span in the spectrum in C), showing that the oscillatory activity is largely captured by the first component, with a smaller alpha component in the second component that is otherwise masked in the electrode activity. C) Power spectral densities for electrode and component signals. D) Spatial spread for components with different peak frequency showing large variation. Each circle corresponds to one component. E) Example spatial pattern coefficients visualized on electrode grids, for high spatial spread (top row), where a component contributes to activity of many electrodes and low spatial spread (bottom row) with a single maximum. In contrast to A), the absolute value is plotted here to better illustrate the spread, regardless of polarity. The electrode with the largest coefficient is marked with a green circle.

positively, such as when oscillations are present for one source, there are also oscillations present in neighboring sources, there is a risk of harmonics canceling out due to spatial mixing. For instance, a temporal shift of $12.5 \mathrm{~ms}$ constitutes a period of $\frac{1}{4} \pi$ for a $10 \mathrm{~Hz}$ alpha, but twice the amount, $\frac{1}{2} \pi$, for a harmonic $20 \mathrm{~Hz}$ beta-rhythm, resulting in waveform changes solely induced by spatial smearing (Schaworonkow and Nikulin, 2019). Therefore, using spatial filtering techniques can be beneficial to explore waveform properties in iEEG recordings.

Fig. 6A shows an example where spatial demixing reveals two different rhythms with varying waveform shape properties. While the frequency spectra (Fig. 6B) of the raw electrode traces activity traces look similar, waveform shape features are masked in the signals because different neighboring (Fig. 6C) rhythmic components with different waveform shape characteristics summate. The estimated components show a differentiation between a triangular rhythm and one that has a spike-wave shape (reminiscent of local field potential traces that can be found for instance in deep layers of macaques (Bollimunta et al., 2008) or mice (Senzai et al., 2019). Differentiation 
bioRxiv preprint doi: https://doi org/10.1101/2021.02.26.433127; this version posted February 28, 2021. The copyright holder for this preprint (which was not certified by peer review) is the author/funder, who has granted bioRxiv a license to display the preprint in perpetuity. It is made available under aCC-BY-NC-ND 4.0 International license.

A
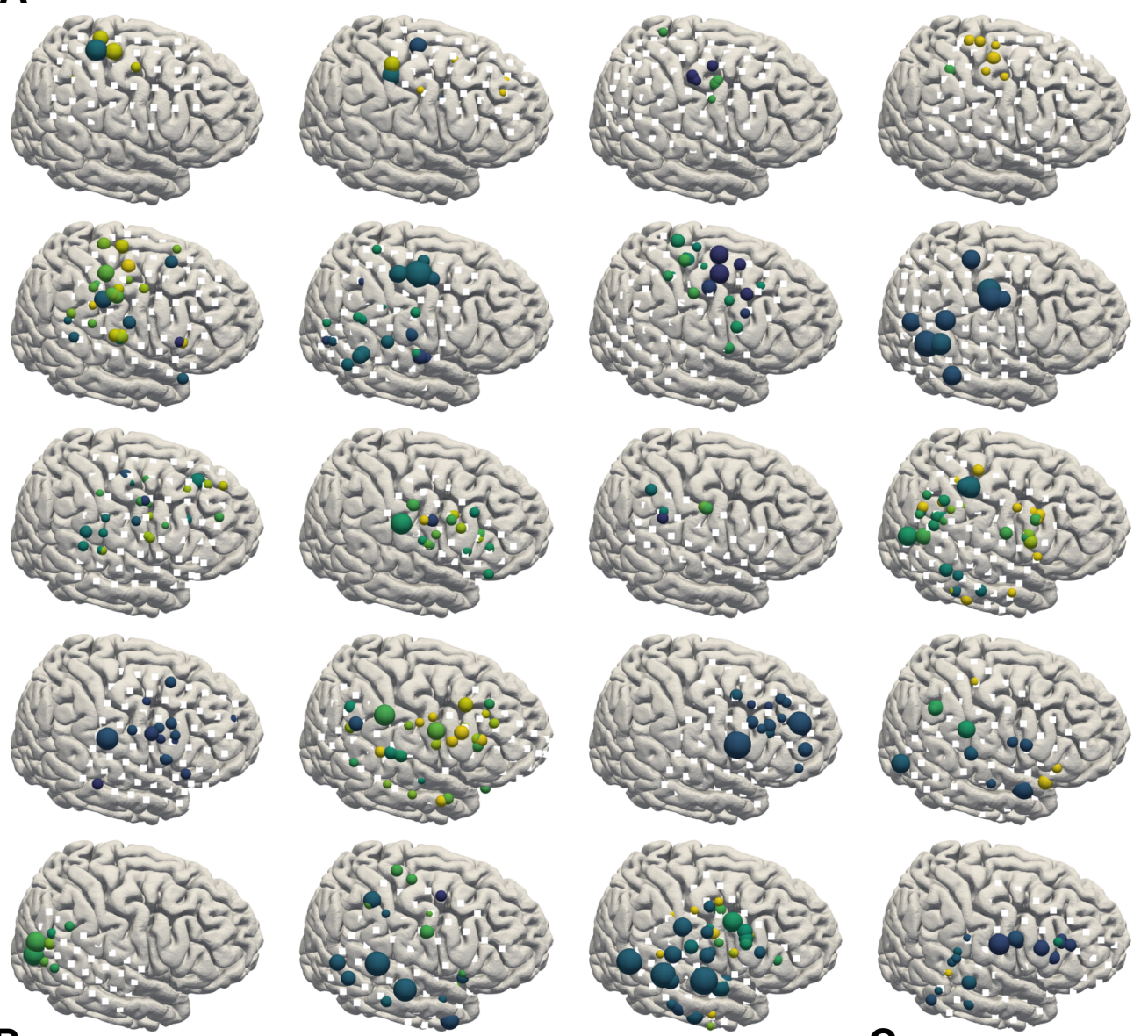

B
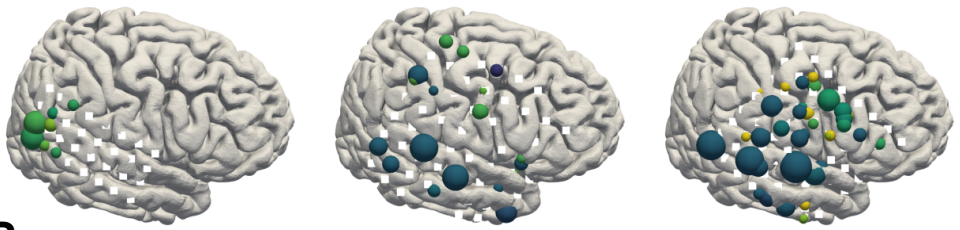

C
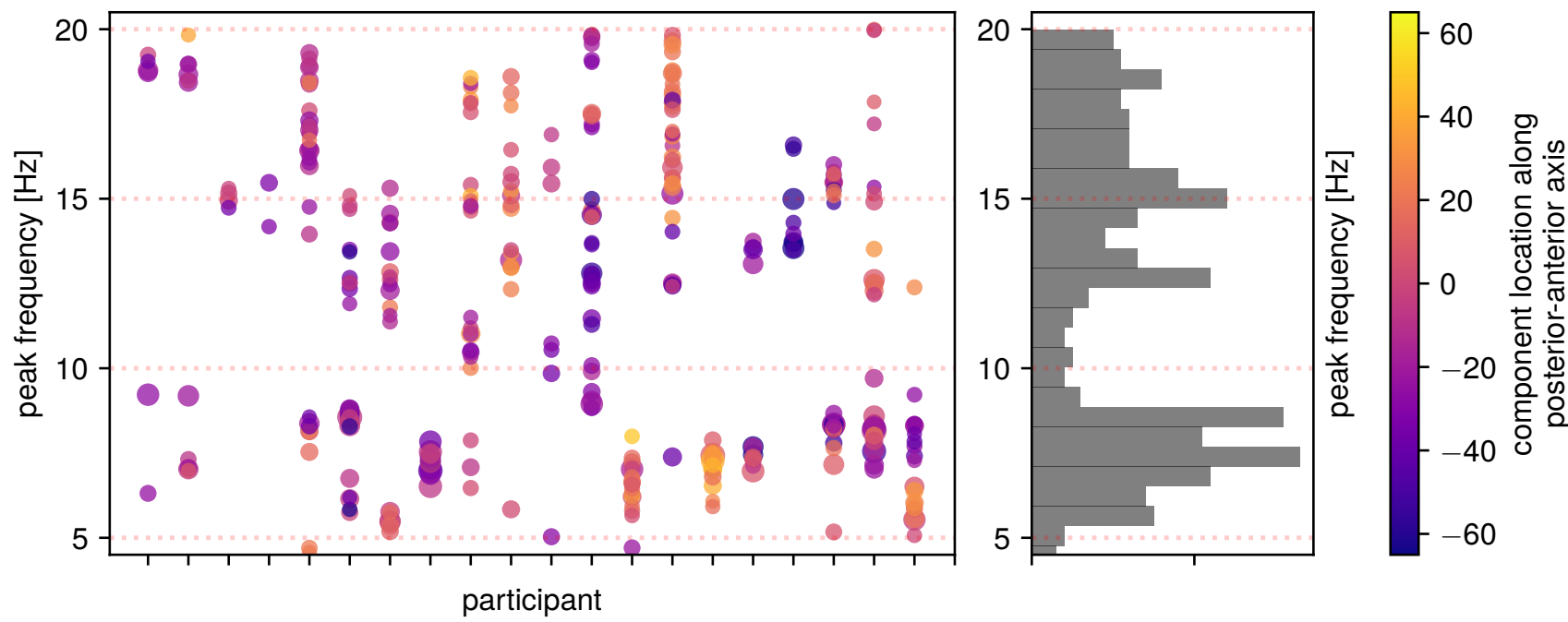

Figure 5: Variability of resting rhythms across the cortex. A) Each subplot shows the location of electrodes (white squares) on a template brain for one individual participant . Each sphere indicates an oscillatory component, with the size indicating 1/f-corrected SNR and the color indicating peak frequency of that component. There is large variability between participants. For improved comparison across participants, all electrodes and rhythm locations were mapped onto the right hemisphere. Participants are ordered according to the mean z-coordinate across the electrode grid, to ease comparison. B) Each component is represented as a circle, with $\mathrm{y}$-position reflecting peak frequency and $\mathrm{x}$-position reflecting participant ID. Color represents position along the posterior-anterior axis, with negative values reflecting most-posterior position. C) Histogram across all participants and components showing a relative lack of detectable $10 \mathrm{~Hz}$ rhythms. 
between different waveforms may be difficult to make on the basis of signals highly impacted by spatial mixing.

Fig. 6D illustrates that many ECoG rhythms in the 5-20 Hz frequency band have a non-sinusoidal waveform shape, which is information that could be taken into account to make inferences about underlying cellular physiology. Here, am peak-trough asymmetry value of 0.2 would mean a peak time of $60 \mathrm{~ms}$ and a trough time of $40 \mathrm{~ms}$ for an oscillation cycle of $100 \mathrm{~ms}$ length (a strong deviation from a 50/50 duty cycle). Non-sinusoidal rhythms can be present in various cortical locations, and in Fig. 6E we show examples of high peak-trough asymmetry in the sensorimotor as well as temporal regions. Of note is that the triangular rhythm visible in Fig. 6A is not asymmetric with respect to duty cycle, but still deviates from a sinusoid by showing sharpness around peaks and troughs. Therefore, construction of measures capturing waveform properties requires careful consideration regarding which aspects to measure, which may be different regarding physiological settings or disease pathologies of interest, e.g., peak-trough asymmetry for the sensorimotor mu-rhythm (Nikulin and Brismar, 2006) or cycle sharpness in Parkinson's disease (Cole et al., 2017).

\subsection{Removing noise with a specific spectral profile using spatial filters}

Another use case of spatial filters that we want to highlight here is in the removal of noise with a specific spectral profile in multichannel data. A prominent noise source in that respect is line noise with a high narrowband spectral peak at e.g., 50 or $60 \mathrm{~Hz}$. Fig. 7 illustrates the removal of noise from a raw ECoG recording that shows high levels of noise at $60 \mathrm{~Hz}$ across the majority of electrodes, as well as narrowband noise at $200 \mathrm{~Hz}$ that is of unknown origin (possibly caused by medical equipment). We estimate spatial filters to maximize SNR first for the $200 \mathrm{~Hz}$ noise, and subsequently project these components out from the raw signal by a linear operation. The signal after removal of noise is shown in Fig. 7B. Fig. 7C shows the signals after applying common average referencing; though the level of noise is attenuated, narrowband noise remains present in the signals. The benefit of using spatial filters to remove noise, as opposed to bandstop filtering, is that spatial filters do not cause distortions in the time domain around the noise frequency. This is especially important because signals in the frequency range of $50-70 \mathrm{~Hz}$ can be highly informative in $\mathrm{ECoG}$, which makes preserving information in this frequency band of particular interest. Another benefit is the computational simplicity, the free parameters here are the peak frequency, the bandwidth around the peak frequency and the number of components to remove. The degree of attenuation can be selected by adjusting the number of noise components to remove, with a larger number yielding greater attenuation at the cost of potentially removing the signal of interest. For line noise removal with spatial filters, the ZapLine toolbox (de Cheveigné, 2020) provides several optimized routines. In ECoG, in addition to line noise, other noise sources can be present, and the flexibility of spatial filters allows for the efficient removal of noise with stationary spectral profiles. The cost is the loss of dimensionality equal to the number of removed components, similar to the effect of applying a common average spatial filter. This loss of dimensionality is not of concern when a high number of electrodes are present, but would not be recommended for a small number of electrodes. While a common average spatial filter may work well if there is a common noise source that is manifesting in all electrode signals, using data-driven spatial filters allows for more flexibility if noise is not present in all signals.

\section{Discussion}

In this article, we highlighted the benefits of using spatial filters for the extraction of neural oscillations in invasive electrophysiological recordings. Applying spatial filters that specifically optimize for oscillatory SNR in iEEG recordings, we assessed presence, spatial spread, variability and waveform shape of iEEG resting rhythms.

\subsection{General benefits of data-driven spatial filtering}

Spatial filters can be used for distinct purposes in the study of neuronal oscillations: to identify rhythms 
A

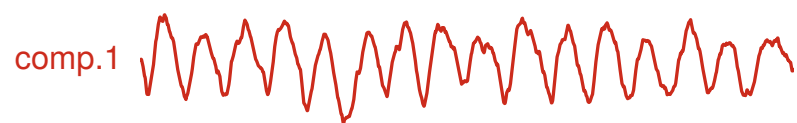

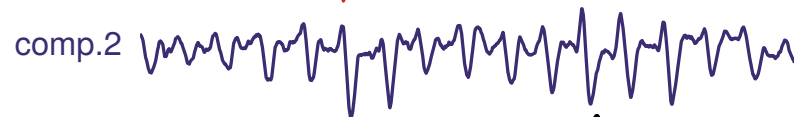
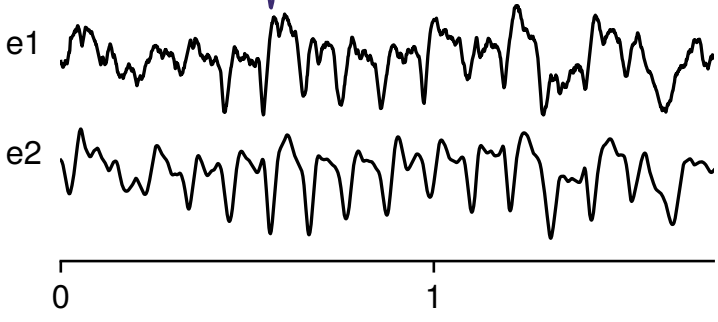

time $[\mathrm{s}]$

D

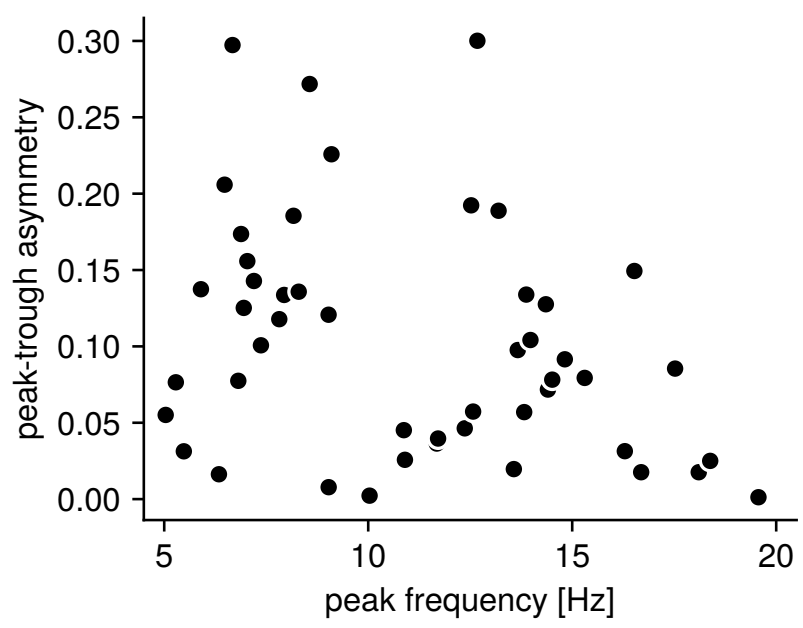

B

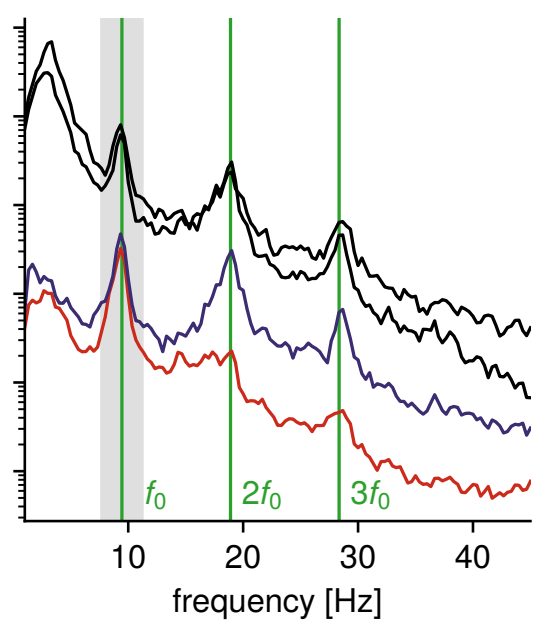

C

$\mathrm{O}^{\circ} \mathrm{O}_{0} \circ \mathrm{O}^{\circ}$

000000

000000 comp.1

e1

(0000

00000

OOOO 00

000000

000000 comp.2

000000 e2

00008

0000008

Figure 6: Waveform shape of intracranial neuronal rhythms. A) Neighboring rhythms with different waveform shape for two electrodes and two components estimated based on alpha band activity. B) Power spectral density for electrodes and components. The presence of harmonic spectral peaks at exact multiples of the alpha peak frequency indicates a non-sinusoidal waveform shape. The gray marked area corresponds to the frequency range defined as a signal for estimation of spatial filters. While both electrode signals show a peak in the beta-band, in component space the sharp beta-harmonic is largely captured by the second component, showing a spike-wave waveform shape, with the first component being a triangular waveform. C) Topographies for the first and second components showing a radial and tangential source distribution (respectively); the electrodes shown as traces in B are marked with green circles. D) Group-level assessment of waveform asymmetry, with intracranial recordings showing considerable peak-trough asymmetry in the waveform (where a peak-trough asymmetry value of 0 is indicating perfect symmetry). E) Peak-trough asymmetry values, plotted across the cortex, larger circles indicate larger SNR. Rhythms with high asymmetry can be found through-out the cortex.

in the frequency band of interest and improve their signal-to-noise ratio, to examine their correlational structure, as well as to denoise data without band-stop filters. On the continuum of using a common average reference spatial filter (potentially capturing mainly non-local activity) to local bipolar spatial filters (with a potentially non-optimal direction of the fixed derivation), the presented spatial filter technique represents a middle ground, extracting signals based on spatial spread as estimated from the data. This procedure results in reduced bias compared to a fixed reference choice.

The presented method belongs to a subclass of spatial filtering techniques that estimate a backwards model using solely the statistical properties of the signals recorded from the electrodes. The benefits of using 
A
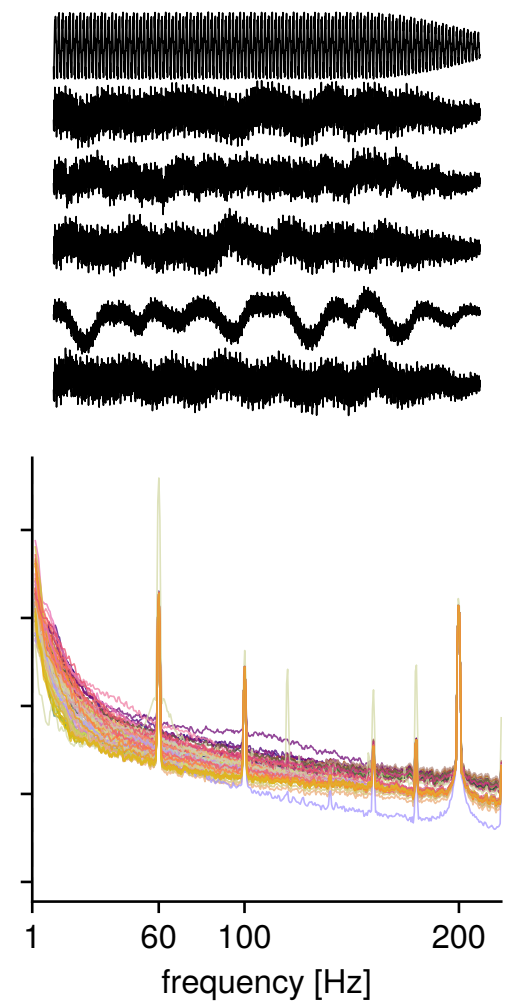

noise removal with SSD

B for 60 and $200 \mathrm{~Hz}$
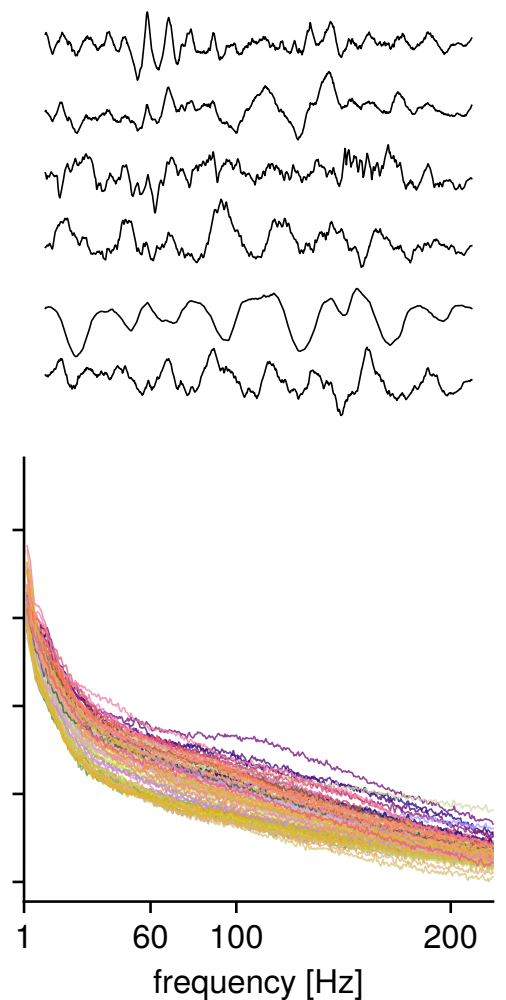
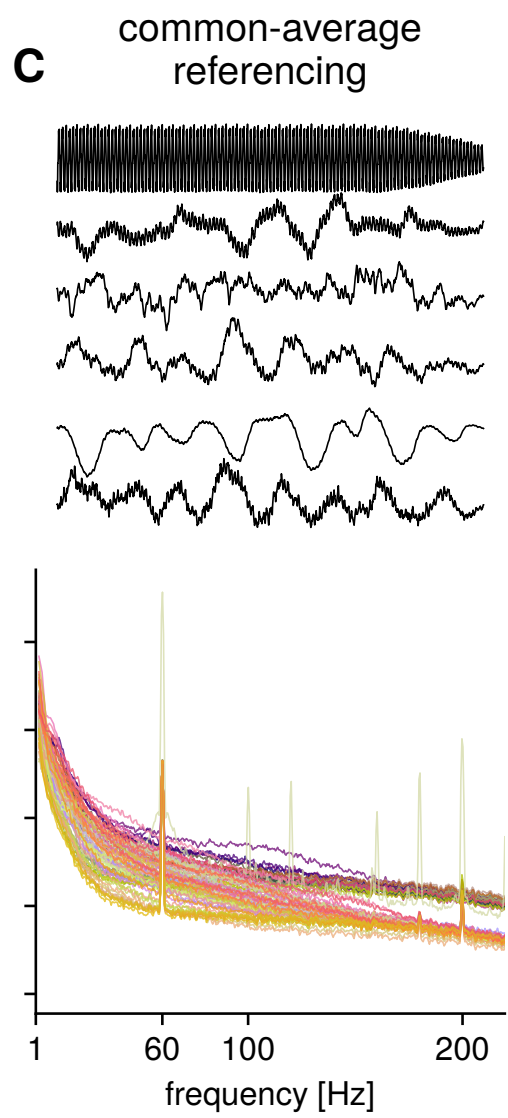

Figure 7: Illustration: removal of noise with spatial filters for ECoG data A) Time series of six electrodes and power spectra for raw ECoG recording for 87 electrodes, color code corresponds to electrode position, with neighboring electrodes having a similar color. B) Time series and power spectra after removal of components maximizing SNR for $60 \mathrm{~Hz}$ and $200 \mathrm{~Hz}$ spectral peaks. Note that there are no band-stop type artefacts in the spectrum since no temporal filtering was performed. C) Time series and power spectra after common average referencing. While the $200 \mathrm{~Hz}$ noise is largely attenuated, $60 \mathrm{~Hz}$ line noise still persists.

statistical approaches like SSD, in contrast to biophysical modelling, is that no anatomical information or biophysical model is required for the estimation of the spatial filters, which strongly reduces the complexity of the procedure. Whenever time series data from multiple electrodes is available, the method can be applied. The electrode locations are only needed for the interpretation of spatial patterns, but the source time series estimation is independent from the localization accuracy of the electrode positions.

The main benefit of SSD for the study of neural oscillations is that information about the signal structure at the frequency band of interest is incorporated, enhancing activity in that band of interest. This procedure is in contrast to independent component analysis or principal component analysis, which both maximize global objectives, which may not be optimal because components in specific frequency bands might only contribute a small amount to global variance. SSD results in an ordering of components according to SNR in the frequency band of interest, which reduces manual inspection and can facilitate data exploration. Additionally, SSD has few parameters and is computationally fast. The signal is defined using a temporal band-pass filter around the frequency of interest. The temporal filter requires a specification of frequency ranges for respective signal and noise contributions, for which the prior values can be derived from electrode power spectra. Even though only narrowband information is used for the estimation of spatial filters, the application on broadband data preserves information beyond these narrow frequency bands, such as waveform shape, as long as they originate from the same spatial location. 
While we chose SSD as a spatial filtering technique for our illustrations, other types of generalized eigenvalue decomposition algorithms are available to solve specific objectives. For enhancing specifically oscillatory SNR, there are variants that maximize the spectral power in a frequency band of interest, compared to the total spectral power (de Cheveigné and Arzounian, 2015), which are benchmarked for EEG in (Cohen, 2017), with demonstrations for MEG/EEG as well as monkey ECoG and optical imaging given in (de Cheveigné and Parra, 2014). For task-based data, Common Spatial Patterns (Koles, 1991) maximizes differences between conditions, for instance to investigate main contributions to task-related modulation. Source Power Correlation analysis (Dähne et al., 2014) maximizes correlation with a target variable, for instance with reaction times. To consider extracting coupled neuronal sources, an extension of SSD termed Nonlinear Interaction Decomposition (Idaji et al., 2020) has been suggested. If specific types of rhythms are of interest, SSD can also be used as a regularization technique before using other analysis methods to limit the analyzed signal to the frequency range of interest, for instance focusing on rhythms in the alpha band (Mahjoory et al., 2017; Haufe et al., 2014a).

\subsection{Benefits of data-driven spatial filtering for $i E E G$ recordings}

While the above listed benefits are general and also hold for applying data-driven spatial filtering for noninvasive electrophysiological signals, there are specific considerations when applying these methods for invasive electrophysiological recordings.

In contrast to noninvasive scalp recordings with standardized EEG electrode caps or sensor arrays in MEG, invasive electrodes are placed according to specific and heterogeneous clinical demands, and therefore do not conform to standardized positions. This complicates the incorporation of anatomical information into analyses. For biophysical source estimation/localization approaches, the accuracy will vary depending on electrode localization accuracy based on postimplantation CT imaging as well as accuracy of the estimated forward model. Reference-choice is also more variable in invasive recordings, with referencing often done to bone or a shank, as well as bipolar or monopolar referencing schemes, which may bias analyses. In the specific case of analyzing neural oscillations, using SSD may be beneficial, since it specifically utilizes information in the frequency band of interest, maximizing SNR in a more flexible way. In addition, nearby electrodes can have different SNR due to the placement of individual electrode contacts on cortical vasculature, which can influence the resulting signal in a frequency-dependent manner when using other common referencing schemes (Bleichner et al., 2011). In addition, SSD allows for the use of information from several grids, or separate but close-by sEEG leads, which can convey improved SNR in contrast to using information from individual leads separately, as in the case of a fixed referencing scheme. The data-driven approach could also be helpful in integrating information from several types of electrodes, for instance in hybrid micro-macro electrode schemes (Chari et al., 2020).

While strong alpha-oscillations tend to dominate in non-invasive recordings, a more variable set of oscillations, with neighboring peak frequencies, is simultaneously detectable in iEEG recordings. To improve the detection of these typically smaller amplitude rhythms, spatial filtering might help to separate them from more dominant frequency rhythms, as SSD attenuates the signal contribution from flanking frequency bands, and therefore the separation of distinct sources in neighboring frequencies becomes possible with SSD.

\subsection{Physiological considerations: spatial spread}

Previous work has identified the degree of spatial correlations across invasively acquired field signals in varying frequency bands (Dubey and Ray, 2019; Kajikawa and Schroeder, 2011; Muller et al., 2016; Katzner et al., 2009), with estimates ranging from $400 \mu \mathrm{m}$ for the local field potential to several millimeters in the case of ECoG. Modeling work by (Lindén et al., 2011) suggests that important consideration is the input correlation of the involved neuronal populations, where spatial spread grows as the degree of input correlations increases. While the spatial spread is limited for high-frequency signals, for low-frequency rhythms the spatial spread exceeds the interelectrode 
distance, which results in the same source contributing to several electrodes.

We showed examples of spatial spread as estimated from spatial patterns, ranging from small to large spread (Fig. 4). The spread for a specific component and possible neighboring signal sources cannot be known a priori and differs across sources and participants. Therefore spatial mixing will potentially obfuscate temporal dynamics of neighboring sources. Data-driven spatial filters can help to separate contributions of different sources onto corresponding electrodes, and spatial pattern coefficients can be used to visualize the composition of electrode activity as a linear combination of different sources. It would be of interest to evaluate whether higher-density ECoG grids with a smaller inter-electrode spacing than analyzed here would result in improved ability to separate rhythms in cortical areas where a large number of independent rhythms with different peak frequencies are present, such as along the sensorimotor strip.

\subsection{Physiological considerations: waveform shape}

The analysis of rhythms using only oscillatory amplitude and frequency discards a lot of potentially valuable physiological information. Using waveform shape measures can enable a more refined look on cellular generation mechanisms and functional relevance of rhythms (Sherman et al., 2016; Krishnakumaran et al., 2021), however the detection of nonsinusoidal features of waveforms requires high SNR, making the analysis of non-sinusoidal waveforms difficult. Because of this, intracranial recordings are well suited for investigations of waveform shape. We show that rhythms as detected in ECoG can be highly non-sinusoidal in a variety of cortical areas. But a potential obstacle is that waveforms can be masked due to spatial mixing of several rhythms. We illustrate that neighboring rhythms, as extracted by spatial filtering, can have different waveform properties that are intermixed at the sensor level (see Fig. 6). It would be informative to relate these to measures from the microscopic scale, e.g., the firing properties of individual neurons in recordings that have both field recordings and single unit spiking data available (Cole and Voytek, 2018). For further analysis of peak frequencies, time domain analysis can help to disen- tangle harmonic from non-harmonic peaks, e.g., a differentiation between non-sinusoidal properties of the sensorimotor mu-rhythm and genuine beta-bursts, a difference that can be obscured by looking at bandpass filtered signals.

\subsection{Physiological considerations: variability of rhythms across participants}

In terms of mapping invasive electrophysiological rhythms, the outlined procedure for investigating dominant rhythms in intracranial data focused on the following methodological considerations: first, electrode activity is always a mixture of many different types of rhythmic and non-rhythmic activity. Therefore, separating putative sources will increase SNR and make it easier to investigate spectral as well as temporal signatures, with e.g., the improved detection of spectral peak frequencies. Not separating sources can result in a "low degree of regional specificity" (Groppe et al., 2013) given that, with a division of channels strictly based on location, volume conduction can lead to a spread of rhythms across regions. Second, the usage of spectral parametrization additionally improves methodological validity in analysis of oscillatory activity through separation from potentially confounding aperiodic activity. Without separation of oscillatory and aperiodic signal contributions, comparing SNR of oscillations in different frequency bands, e.g., for neighboring theta- and alpha band rhythms would require for instance signal whitening, the outcome of which depends on the frequency range used for normalization. By requiring a minimum height of a spectral peak exceeding the aperiodic 1/f-contribution, we ensure to capture oscillatory dynamics.

Equipped with these considerations, we observed that there is high variability in measurable rhythms for individual participants, with for instance no or only weak rhythms in the canonical alpha-frequency range across the sensorimotor cortex, as also observed by (Groppe et al., 2013). The large degree of variability puts the spotlight on the common practice of electrode pooling, or combining all electrodes from all participants for analysis, which might inflate false positives due to the contribution of a high number of significant electrodes from a single participant. In light of the observed variability as well as a variable number 
of electrodes present for each participant, hierarchical models and bootstrapping approaches (Saravanan et al., 2020) should be considered in the analysis of intracranial data, to reduce the risk of only a small fraction of participants showing the effects in the group average.

\subsection{Limitations}

As a general limitation, the estimation of a backward model will never achieve perfect accuracy because dozens of electrodes are not enough to capture the thousands of underlying sources of neuronal activity. Specific limitations of an approach for estimating spatial filters utilizing eigenvalue decomposition are detailed below.

First, there is no automatic one-to-one mapping from estimated components onto physiological entities (but neither can this be done from electrode-based activity). In the case of distinct, but highly co-fluctuating neuronal sources, they will not necessarily be separable on the basis of their covariance. An indication of this are spatial patterns that deviate from the spatial pattern expected for a dipolar source, e.g., by showing several spatially distributed maxima. Approaches based on eigenvalue decomposition (including PCA and ICA) will return as many components as there are electrodes, but not all components will be physiology meaningful in the sense of representing a neuronal source in the frequency band of interest. In deciding how many components to keep for analysis, the following aspects should be considered when using SSD: Inspecting the relative SNR with aid of the power spectrum is crucial and is simplified because the components are ordered according to SNR in the frequency band of interest. Components without a spectral peak in the frequency band of interest should not be considered when talking about neuronal oscillations in that specific frequency band. The spatial patterns should be inspected for determining the local focus of the generating source. In addition, bootstrapping approaches based on surrogate data have been suggested to estimate the number of components to retain (Zuure et al., 2020).

Further, the estimated spatial filters are invariant with respect to signal polarity, i.e., the sign cannot be uniquely determined. Therefore depending on the choice of parameters, the spatial filter can result in a polarity-inverted signal. Alignment of spatially filtered signals can for instance be accomplished according to the sign of the electrode signals, and is straightforward in the case of radially orientated components. In the case of tangentially orientated components, with negative and positive contributions to activity recorded on electrodes, alignment can be made by incorporating knowledge about physiology. Features derived from physiology can include waveform shape, as in the case of the arc-shaped mu-rhythm, or polarity of evoked responses.

Finally, the underlying assumption here is a linear model, and the estimated spatial filters are not dependent on time. This assumption might insufficiently capture traveling wave phenomena, for instance. Propagating activity with high velocity will impact very sharp waveforms, as for electrodes linearly combined with a slight offset a sharp trough will result in a less sharp trough for the component due to timeindependent linear combination. It would be of interest for future directions to take wave propagation into account when estimating neuronal oscillatory sources (Kuznetsova et al., 2020; Hindriks, 2020).

\subsection{Conclusion}

Invasive electrophysiological recordings allow for high spatial and temporal resolution investigations into the functional role of the diversity of neuronal oscillations that are present across the cortex. Different types of oscillations can be seen, showing specific spatial distributions, peak frequencies, waveform shapes and functional modulation, all of which indicate diverse underlying physiology. The spatial and temporal overlap of these rhythms makes the measurement of these different features difficult when only using data derived from single electrodes. Here, we argue that the richness of the data can be better explored when applying data-driven spatial filters, which use multichannel information to specifically enhance the signal-to-noise ratio of oscillations, and therefore improve our ability to study them. This, in turn, helps bridge scales between invasive intracranial measurements and noninvasive, macroscale scalp measurements. 


\section{Funding}

This work was supported by the Whitehall Foundation (2017-12-73), the NIH National Institute of General Medical Sciences grant R01GM134363-01, and a UC San Diego Halıcıoğlu Data Science Institute Fellowship.

\section{Competing interests}

The authors declare no competing interests.

\section{References}

G. Arnulfo, J. Hirvonen, L. Nobili, S. Palva, J. M. Palva, Phase and amplitude correlations in resting-state activity in human stereotactical EEG recordings, NeuroImage 112 (2015) 114 127.

M. G. Bleichner, M. J. Vansteensel, G. M. Huiskamp, D. Hermes, E. J. Aarnoutse, C. H. Ferrier, N. F. Ramsey, The effects of blood vessels on electrocorticography, Journal of Neural Engineering 8 (4) (2011) 044002.

A. Bollimunta, Y. Chen, C. E. Schroeder, M. Ding, Neuronal Mechanisms of Cortical Alpha Oscillations in AwakeBehaving Macaques, Journal of Neuroscience 28 (40) (2008) 9976-9988.

G. Buzsáki, C. A. Anastassiou, C. Koch, The origin of extracellular fields and currents - EEG, ECoG, LFP and spikes, Nature Reviews Neuroscience 13 (6) (2012) 407-420.

A. Chari, R. C. Thornton, M. M. Tisdall, R. C. Scott, Microelectrode recordings in human epilepsy: a case for clinical translation, Brain Communications 2 (2) (2020) fcaa082.

C. Chintaluri, D. K. Wójcik, A novel method for spatial source localization using ECoG and SEEG recordings in human epilepsy patients, BMC Neuroscience 16 (S1) (2015) P286.

M. X. Cohen, Comparison of linear spatial filters for identifying oscillatory activity in multichannel data, Journal of Neuroscience Methods 278 (2017) 1-12.

S. Cole, B. Voytek, Hippocampal theta bursting and waveform shape reflect CA1 spiking patterns, preprint, Neuroscience, 10.1101/452987, 2018.

S. Cole, B. Voytek, Cycle-by-cycle analysis of neural oscillations, Journal of Neurophysiology 122 (2) (2019) 849-861.

S. R. Cole, R. van der Meij, E. J. Peterson, C. de Hemptinne, P. A. Starr, B. Voytek, Nonsinusoidal Beta Oscillations Reflect Cortical Pathophysiology in Parkinson's Disease, The Journal of Neuroscience 37 (18) (2017) 4830-4840.

S. R. Cole, B. Voytek, Brain Oscillations and the Importance of Waveform Shape, Trends in Cognitive Sciences 21 (2) (2017) 137-149.

N. E. Crone, D. L. Miglioretti, B. Gordon, R. P. Lesser, Functional mapping of human sensorimotor cortex with electrocorticographic spectral analysis. II. Event-related synchronization in the gamma band., Brain 121 (12) (1998a) 2301-2315.
N. E. Crone, D. L. Miglioretti, B. Gordon, J. M. Sieracki, M. T. Wilson, S. Uematsu, R. P. Lesser, Functional mapping of human sensorimotor cortex with electrocorticographic spectral analysis. I. Alpha and beta event- related desynchronization, Brain 121 (12) (1998b) 2271-99.

A. de Cheveigné, ZapLine: A simple and effective method to remove power line artifacts, NeuroImage 207 (2020) 116356.

A. de Cheveigné, D. Arzounian, Scanning for oscillations, Journal of Neural Engineering 12 (6) (2015) 066020.

A. de Cheveigné, L. C. Parra, Joint decorrelation, a versatile tool for multichannel data analysis, NeuroImage 98 (2014) 487-505.

T. Donoghue, M. Haller, E. J. Peterson, P. Varma, P. Sebastian, R. Gao, T. Noto, A. H. Lara, J. D. Wallis, R. T. Knight, A. Shestyuk, B. Voytek, Parameterizing neural power spectra into periodic and aperiodic components, Nature Neuroscience 23 (12) (2020) 1655-1665.

A. Dubey, S. Ray, Cortical Electrocorticogram (ECoG) Is a Local Signal, The Journal of Neuroscience 39 (22) (2019) 4299-4311.

S. Dähne, V. V. Nikulin, D. Ramírez, P. J. Schreier, K.-R. Müller, S. Haufe, Finding brain oscillations with power dependencies in neuroimaging data, NeuroImage 96 (2014) 334-348.

M. Dümpelmann, T. Ball, A. Schulze-Bonhage, sLORETA allows reliable distributed source reconstruction based on subdural strip and grid recordings, Human Brain Mapping 33 (5) (2012) 1172-1188.

A. K. Engel, C. K. E. Moll, I. Fried, G. A. Ojemann, Invasive recordings from the human brain: clinical insights and beyond, Nature Reviews Neuroscience 6 (1) (2005) 35-47.

M. Fahimi Hnazaee, B. Wittevrongel, E. Khachatryan, A. Libert, E. Carrette, I. Dauwe, A. Meurs, P. Boon, D. Van Roost, M. M. Van Hulle, Localization of deep brain activity with scalp and subdural EEG, NeuroImage 223 (2020) 117344.

A. Flinker, E. Chang, N. Barbaro, M. Berger, R. Knight, Subcentimeter language organization in the human temporal lobe, Brain and Language 117 (3) (2011) 103-109.

B. Frauscher, N. von Ellenrieder, R. Zelmann, I. Doležalová, L. Minotti, A. Olivier, J. Hall, D. Hoffmann, D. K. Nguyen, P. Kahane, F. Dubeau, J. Gotman, Atlas of the normal intracranial electroencephalogram: neurophysiological awake activity in different cortical areas, Brain 141 (4) (2018) 11301144.

M. Fuchs, M. Wagner, J. Kastner, Development of Volume Conductor and Source Models to Localize Epileptic Foci:, Journal of Clinical Neurophysiology 24 (2) (2007) 101-119.

A. Gramfort, M. Luessi, E. Larson, D. A. Engemann, D. Strohmeier, C. Brodbeck, R. Goj, M. Jas, T. Brooks, L. Parkkonen, M. Hämäläinen, MEG and EEG data analysis with MNE-Python, Frontiers in Neuroscience 7.

H. Gray, Antomy of the human body, Annals of surgery 68 (5) (1918) 564-566.

D. M. Groppe, S. Bickel, C. J. Keller, S. K. Jain, S. T. Hwang, C. Harden, A. D. Mehta, Dominant frequencies of resting human brain activity as measured by the electrocorticogram, NeuroImage 79 (2013) 223-233. 
bioRxiv preprint doi: https://doi.org/10.1101/2021.02.26.433127; this version posted February 28, 2021. The copyright holder for this preprint (which was not certified by peer review) is the author/funder, who has granted bioRxiv a license to display the preprint in perpetuity. It is made available under aCC-BY-NC-ND 4.0 International license.

M. Halgren, I. Ulbert, H. Bastuji, D. Fabó, L. Erôss, M. Rey, O. Devinsky, W. K. Doyle, R. Mak-McCully, E. Halgren, L. Wittner, P. Chauvel, G. Heit, E. Eskandar, A. Mandell, S. S. Cash, The generation and propagation of the human alpha rhythm, Proceedings of the National Academy of Sciences 116 (47) (2019) 23772-23782.

S. Haufe, S. Dähne, V. V. Nikulin, Dimensionality reduction for the analysis of brain oscillations, NeuroImage 101 (2014a) 583-97.

S. Haufe, F. Meinecke, K. Görgen, S. Dähne, J.-D. Haynes, B. Blankertz, F. Bießmann, On the interpretation of weight vectors of linear models in multivariate neuroimaging, NeuroImage 87 (2014b) 96-110.

R. Hindriks, A methodological framework for inverse-modeling of propagating cortical activity using MEG/EEG, NeuroImage 223 (2020) 117345.

R. Hindriks, C. Micheli, C. Bosman, R. Oostenveld, C. Lewis, D. Mantini, P. Fries, G. Deco, Source-reconstruction of the sensorimotor network from resting-state macaque electrocorticography, NeuroImage 181 (2018) 347-358.

J. F. Hipp, D. J. Hawellek, M. Corbetta, M. Siegel, A. K. Engel, Large-scale cortical correlation structure of spontaneous oscillatory activity, Nature Neuroscience 15 (6) (2012) 884 890.

S. Hu, M. Stead, G. Worrell, Automatic Identification and Removal of Scalp Reference Signal for Intracranial EEGs Based on Independent Component Analysis, IEEE Transactions on Biomedical Engineering 54 (9) (2007) 1560-1572.

M. J. Idaji, K.-R. Müller, G. Nolte, B. Maess, A. Villringer, V. V. Nikulin, Nonlinear interaction decomposition (NID): A method for separation of cross-frequency coupled sources in human brain, NeuroImage 211 (2020) 116599.

J. Jacobs, M. J. Kahana, Direct brain recordings fuel advances in cognitive electrophysiology, Trends in Cognitive Sciences 14 (4) (2010) 162-171.

M. Jas, E. Larson, D. A. Engemann, J. Leppäkangas, S. Taulu, M. Hämäläinen, A. Gramfort, A Reproducible MEG/EEG Group Study With the MNE Software: Recommendations, Quality Assessments, and Good Practices, Frontiers in Neuroscience 12 (2018) 530.

H. Jasper, W. Penfield, Electrocorticograms in man: Effect of voluntary movement upon the electrical activity of the precentral gyrus, Archiv für Psychiatrie und Nervenkrankheiten 183 (1-2) (1949) 163-174.

Y. Kajikawa, C. Schroeder, How Local Is the Local Field Potential?, Neuron 72 (5) (2011) 847-858.

S. Katzner, I. Nauhaus, A. Benucci, V. Bonin, D. L. Ringach, M. Carandini, Local Origin of Field Potentials in Visual Cortex, Neuron 61 (1) (2009) 35-41.

$\mathrm{Z}$. Koles, The quantitative extraction and topographic mapping of the abnormal components in the clinical EEG, Electroencephalography and Clinical Neurophysiology 79 (6) (1991) 440-447.

R. Krishnakumaran, M. Raees, S. Ray, Shape analysis of gamma rhythm supports a superlinear inhibitory regime in an inhibition-stabilized network, preprint, Neuroscience, 2021.
A. Kuznetsova, M. Lebedev, A. Ossadtchi, Local propagation dynamics of MEG interictal spikes: source reconstruction with traveling wave priors, preprint, Neuroscience, 2020.

G. Li, S. Jiang, S. E. Paraskevopoulou, M. Wang, Y. Xu, Z. Wu, L. Chen, D. Zhang, G. Schalk, Optimal referencing for stereoelectroencephalographic (SEEG) recordings, NeuroImage 183 (2018) 327-335.

H. Lindén, T. Tetzlaff, T. Potjans, K. Pettersen, S. Grün, M. Diesmann, G. Einevoll, Modeling the Spatial Reach of the LFP, Neuron 72 (5) (2011) 859-872.

Y. Liu, W. G. Coon, A. d. Pesters, P. Brunner, G. Schalk, The effects of spatial filtering and artifacts on electrocorticographic signals, Journal of Neural Engineering 12 (5) (2015) 056008.

K. Mahjoory, V. V. Nikulin, L. Botrel, K. Linkenkaer-Hansen, M. M. Fato, S. Haufe, Consistency of EEG source localization and connectivity estimates, NeuroImage 152 (2017) 590-601.

S. Michelmann, M. S. Treder, B. Griffiths, C. Kerrén, F. Roux, M. Wimber, D. Rollings, V. Sawlani, R. Chelvarajah, S. Gollwitzer, G. Kreiselmeyer, H. Hamer, H. Bowman, B. Staresina, S. Hanslmayr, Data-driven re-referencing of intracranial EEG based on independent component analysis (ICA), Journal of Neuroscience Methods 307 (2018) 125-137.

K. J. Miller, A library of human electrocorticographic data and analyses, Nature Human Behaviour 3 (11) (2019) 1225-1235.

K. J. Miller, G. Schalk, D. Hermes, J. G. Ojemann, R. P. N. Rao, Spontaneous Decoding of the Timing and Content of Human Object Perception from Cortical Surface Recordings Reveals Complementary Information in the Event-Related Potential and Broadband Spectral Change, PLOS Computational Biology 12 (1) (2016) e1004660.

K. J. Miller, L. B. Sorensen, J. G. Ojemann, M. den Nijs, PowerLaw Scaling in the Brain Surface Electric Potential, PLoS Computational Biology 5 (12) (2009) e1000609.

D. J. Mitchell, N. McNaughton, D. Flanagan, I. J. Kirk, Frontalmidline theta from the perspective of hippocampal "theta", Progress in Neurobiology 86 (3) (2008) 156-185.

L. Muller, L. S. Hamilton, E. Edwards, K. E. Bouchard, E. F. Chang, Spatial resolution dependence on spectral frequency in human speech cortex electrocorticography, Journal of Neural Engineering 13 (5) (2016) 056013.

V. Nikulin, T. Brismar, Phase synchronization between alpha and beta oscillations in the human electroencephalogram, Neuroscience 137 (2) (2006) 647-657.

V. V. Nikulin, G. Nolte, G. Curio, A novel method for reliable and fast extraction of neuronal EEG/MEG oscillations on the basis of spatio-spectral decomposition, NeuroImage 55 (4) (2011) 1528-1535.

L. C. Parra, C. D. Spence, A. D. Gerson, P. Sajda, Recipes for the linear analysis of EEG, NeuroImage 28 (2) (2005) 326-341.

A. Pascarella, C. Todaro, M. Clerc, T. Serre, M. Piana, Source modeling of ElectroCorticoGraphy (ECoG) data: Stability analysis and spatial filtering, Journal of Neuroscience Methods 263 (2016) 134-144.

V. Saravanan, G. J. Berman, S. J. Sober, Application of the hierarchical bootstrap to multi-level data in neuroscience, 2020. 
N. Schaworonkow, V. V. Nikulin, Spatial neuronal synchronization and the waveform of oscillations: Implications for EEG and MEG, PLOS Computational Biology 15 (5) (2019) e1007055.

N. Schaworonkow, J. Triesch, U. Ziemann, C. Zrenner, EEGtriggered TMS reveals stronger brain state-dependent modulation of motor evoked potentials at weaker stimulation intensities, Brain Stimulation 12 (1) (2019) 110-118.

Y. Senzai, A. Fernandez-Ruiz, G. Buzsáki, Layer-Specific Physiological Features and Interlaminar Interactions in the Primary Visual Cortex of the Mouse, Neuron 101 (3) (2019) 500513.e5.

M. A. Sherman, S. Lee, R. Law, S. Haegens, C. A. Thorn, M. S. Hämäläinen, C. I. Moore, S. R. Jones, Neural mechanisms of transient neocortical beta rhythms: Converging evidence from humans, computational modeling, monkeys, and mice, Proceedings of the National Academy of Sciences 113 (33) (2016) E4885-E4894.

A. Stolk, L. Brinkman, M. J. Vansteensel, E. Aarnoutse, F. S. Leijten, C. H. Dijkerman, R. T. Knight, F. P. de Lange, I. Toni, Electrocorticographic dissociation of alpha and beta rhythmic activity in the human sensorimotor system, eLife 8 (2019) e48065.

N. Suthana, I. Fried, Percepts to recollections: insights from single neuron recordings in the human brain, Trends in Cognitive Sciences 16 (8) (2012) 427-436.

D. Whitmer, G. Worrell, M. Stead, I. K. Lee, S. Makeig, Utility of Independent Component Analysis for Interpretation of Intracranial EEG, Frontiers in Human Neuroscience 4.

M. B. Zuure, L. B. Hinkley, P. H. Tiesinga, S. S. Nagarajan, M. X. Cohen, Multiple midfrontal thetas revealed by source separation of simultaneous MEG and EEG, Journal of Neuroscience 40 (40) (2020) 7702-7713. 